\title{
Solving Large-Scale Multi-Objective Optimization Problems with Sparse Optimal Solutions via Unsupervised Neural Networks
}

\author{
Ye Tian, Chang Lu, Xingyi Zhang, Senior Member, IEEE, Kay Chen Tan, Fellow, IEEE, \\ and Yaochu Jin, Fellow, IEEE
}

\begin{abstract}
Due to the curse of dimensionality of search space, it is extremely difficult for evolutionary algorithms to approximate the optimal solutions of large-scale multiobjective optimization problems (LMOPs) by using a limited budget of evaluations. If the Pareto optimal subspace is approximated during the evolutionary process, the search space can be reduced and the difficulty encountered by evolutionary algorithms can be highly alleviated. Following the above idea, this paper proposes an evolutionary algorithm to solve sparse LMOPs by learning the Pareto optimal subspace. The proposed algorithm uses two unsupervised neural networks, a restricted Boltzmann machine and a denoising autoencoder to learn a sparse distribution and a compact representation of the decision variables, where the combination of the learnt sparse distribution and compact representation is regarded as an approximation of the Pareto optimal subspace. The genetic operators are conducted in the learnt subspace, and the resultant offspring solutions then can be mapped back to the original search space by the two neural networks. According to the experimental results on eight benchmark problems and eight real-world problems, the proposed algorithm can effectively solve sparse LMOPs with 10,000 decision variables by only 100,000 evaluations.
\end{abstract}

Manuscript received -. This work was supported in part by the National Key Research and Development Project under Grant 2018AAA0100105, in part by the National Natural Science Foundation of China under Grant 61672033, Grant 61822301, Grant 61876123, Grant 61906001, and Grant U1804262, in part by the Hong Kong Scholars Program under Grant XJ2019035, in part by the Anhui Provincial Natural Science Foundation under Grant 1808085J06 and Grant 1908085QF271, in part by the State Key Laboratory of Synthetical Automation for Process Industries under Grant PAL-N201805, and in part by the Research Grants Council of the Hong Kong Special Administrative Region, China under Grant CityU11202418 and Grant CityU11209219, (Corresponding author: Xingyi Zhang.)

Y. Tian is with the Key Laboratory of Intelligent Computing and Signal Processing of Ministry of Education, Institutes of Physical Science and Information Technology, Anhui University, Hefei 230601, China, and also with the Department of Computer Science, City University of Hong Kong, Kowloon Tong, Hong Kong SAR (email: field910921@gmail.com).

C. Lu and X. Zhang are with the Key Laboratory of Intelligent Computing and Signal Processing of Ministry of Education, School of Computer Science and Technology, Anhui University, Hefei 230601, China (email: lucy_lc@outlook.com; xyzhanghust@gmail.com).

K. C. Tan is with the Department of Computer Science, City University of Hong Kong, Kowloon Tong, Hong Kong SAR (email: kaytan@cityu.edu.hk).

Y. Jin is with the Department of Computer Science, University of Surrey, Guildford, Surrey, GU2 7XH, U.K., and also with the Department of Computer Science and Engineering, Southern University of Science and Technology, Shenzhen 518055, China (email: yaochu.jin@surrey.ac.uk).
Index Terms-Large-scale multi-objective optimization, sparse Pareto optimal solutions, Pareto optimal subspace, restricted Boltzmann machine, denoising autoencoder

\section{INTRODUCTION}

I $\mathrm{N}$ the era of big data, there exists plenty of complicated data in many research fields and real-world applications, which raises a variety of optimization problems having multiple objectives and a large number of decision variables [1]-[3]. These large-scale multiobjective optimization problems (LMOPs) present a huge search space that grows exponentially with the number of decision variables, posing stiff challenges for evolutionary algorithms to efficiently approximate the Pareto optimal solutions [4]. To address the inevitable "curse of dimensionality", some multi-objective evolutionary algorithms (MOEAs) have been tailored for LMOPs, which are mainly based on the following two ideas.

The first idea for solving LMOPs is decision variable decomposition, which adopts the divide-and-conquer strategy that divides the decision variables into several groups randomly or heuristically, and optimizes each group of decision variables separately. For example, NSCCGA [5] relates each decision variable to a subpopulation, then optimizes each subpopulation by NSGAII [6]. MOEA/DVA [7] divides the decision variables into position variables, distance variables, and mixed variables, and further divides the distance variables according to their interactions on the objective functions. MOEA/DVA first optimizes each group of distance variables until the population converges, then fine-tunes all the decision variables for better diversity. LMEA [8] clusters the decision variables into convergence-related variables and diversity-related variables, and iteratively optimizes each type of variables by different strategies.

The second idea for solving LMOPs is problem transformation, which aims to convert the original LMOP into a small-scale problem, so that it can be handled by general optimizers. Different from traditional decomposition based MOEAs (e.g., those based on hierarchical decomposition [9] or Minkowski distance [10]) using a set of weights to transfer multi-objective optimization into single-objective optimization, WOF [11] uses a set 
of weights to alter the decision variables, where each weight is related to multiple decision variables and the number of weights is much smaller than the number of decision variables. As a result, a small-scale problem can be established by considering the weights as variables to be optimized. LSMOF [12] defines two reference directions on a solution in search space, and searches for better solutions by moving the solutions along the reference directions. In other words, better solutions can be found by optimizing only two weights, where each weight determines the location of the solution in a reference direction.

In spite of the promising performance of these MOEAs on some LMOPs, most of them are shown to be of low efficiency or effectiveness [13]. For the decision variable decomposition based MOEAs, a large number of function evaluations are required for detecting the interactions between decision variables, and the detecting results are probably inaccurate on functions with complicated landscapes [8]. The problem transformation based MOEAs are more vulnerable to getting trapped in local optimums due to the loss of diversified search directions [12], though they can quickly find well-converged solutions by optimizing a small-scale problem.

According to the Karush-Kuhn-Tucker condition [14], the Pareto optimal solutions of an LMOP constitute an $(M-1)$-dimensional piecewise continuous manifold, where $M$ is the number of objectives and usually much smaller than the number of decision variables. That is, all the Pareto optimal solution can fill a $(M-1)$-dimensional Pareto optimal subspace, which accounts for a tiny proportion of the original $D$-dimensional search space since $M \ll D$. Therefore, the original search space is reducible if some quasi-optimal solutions have been found, and the difficulty of LMOPs can be highly alleviated. In fact, this regularity property has been essential for many estimation of distribution algorithms [15], [16]. Nevertheless, these algorithms still encounter difficulties in solving LMOPs [13], [16], since the interactions between decision variables are so complex that it is difficult to learn the accurate Pareto optimal subspace.

In this paper, we propose a Pareto optimal subspace learning based evolutionary algorithm for solving the LMOPs whose Pareto optimal solutions are sparse, i.e., most decision variables of the Pareto optimal solutions are zero. Such LMOPs widely exist in many real-world applications [17]-[19], but there does not exist any MOEA tailored for them so far. Specifically, the proposed algorithm, termed MOEA/PSL, includes the following two main contributions:

1) The restricted Boltzmann machine (RBM) [20] and denoising autoencoder (DAE) [21] are adopted in the proposed MOEA/PSL to learn the Pareto optimal subspace. At the beginning of each generation, the decision variables of the non-dominated solutions are used to train the two neural networks, where the RBM is used to learn a sparse distribution of the decision variables and the DAE is used to learn a compact representation. The combination of the sparse distribution and compact representation is regarded as an approximation of the Pareto optimal subspace, where genetic operators are conducted in the learnt subspace instead of the original search space. Therefore, the original search space is highly reduced.

2) A parameter adaptation strategy is designed to automatically determine the parameters in Pareto optimal subspace learning. On one hand, the size of hidden layers of the two neural networks is estimated according to the sparsity of the nondominated solutions. On the other hand, the ratio of offspring solutions generated in the learnt subspace is dynamically adjusted according to the number of successful offspring solutions generated at the previous generations. This way, the proposed algorithm can adapt to different sparse LMOPs without any predefined parameter.

The rest of this paper is organized as follows. In Section II, an introduction to the sparse LMOPs in realworld applications is given, and existing Pareto optimal subspace learning approaches are reviewed. Section III presents the proposed MOEA/PSL in detail. In Section IV, the experimental results are presented and analyzed. Finally, conclusions are drawn and future work is outlined in Section V.

\section{RELATED WORK}

\section{A. Sparse LMOPs in Real-World Applications}

As listed in Table I, sparse LMOPs widely exist in many fields including machine learning, network science, software engineering, signal processing, data mining, economics, and so on. As presented in the table, these sparse LMOPs contain 10 to $10^{5}$ binary or real variables. The binary variable based LMOPs aim to select or delete a small number of elements from a large candidate set for optimizing specific objectives. For example, the goals of feature selection [17] and instance selection [24] are to select a few features and instances from the training set for the minimum classification error, respectively. Besides, the real variable based LMOPs aim to find the optimal values of a set of parameters, such as the weights in neural network training [37] and the original signal in sparse signal reconstruction [18].

A significant feature of these problems is that their Pareto optimal solutions are sparse. Most of these problems consider the sparsity of solution as an objective to be optimized (e.g., the number of selected features in feature selection, the model complexity in neural network training, and the sparsity of the signal in sparse signal reconstruction), hence the Pareto optimal solutions are sparse. Although some other problems do not explicitly optimize the sparsity of solution, the Pareto optimal solutions are also very sparse due to their objectives. For instance, the community detection problem [38] aims to identify the community centers from all the nodes, 
TABLE I

17 SPARSE LMOPS IN REAL-WORLD APPLICATIONS.

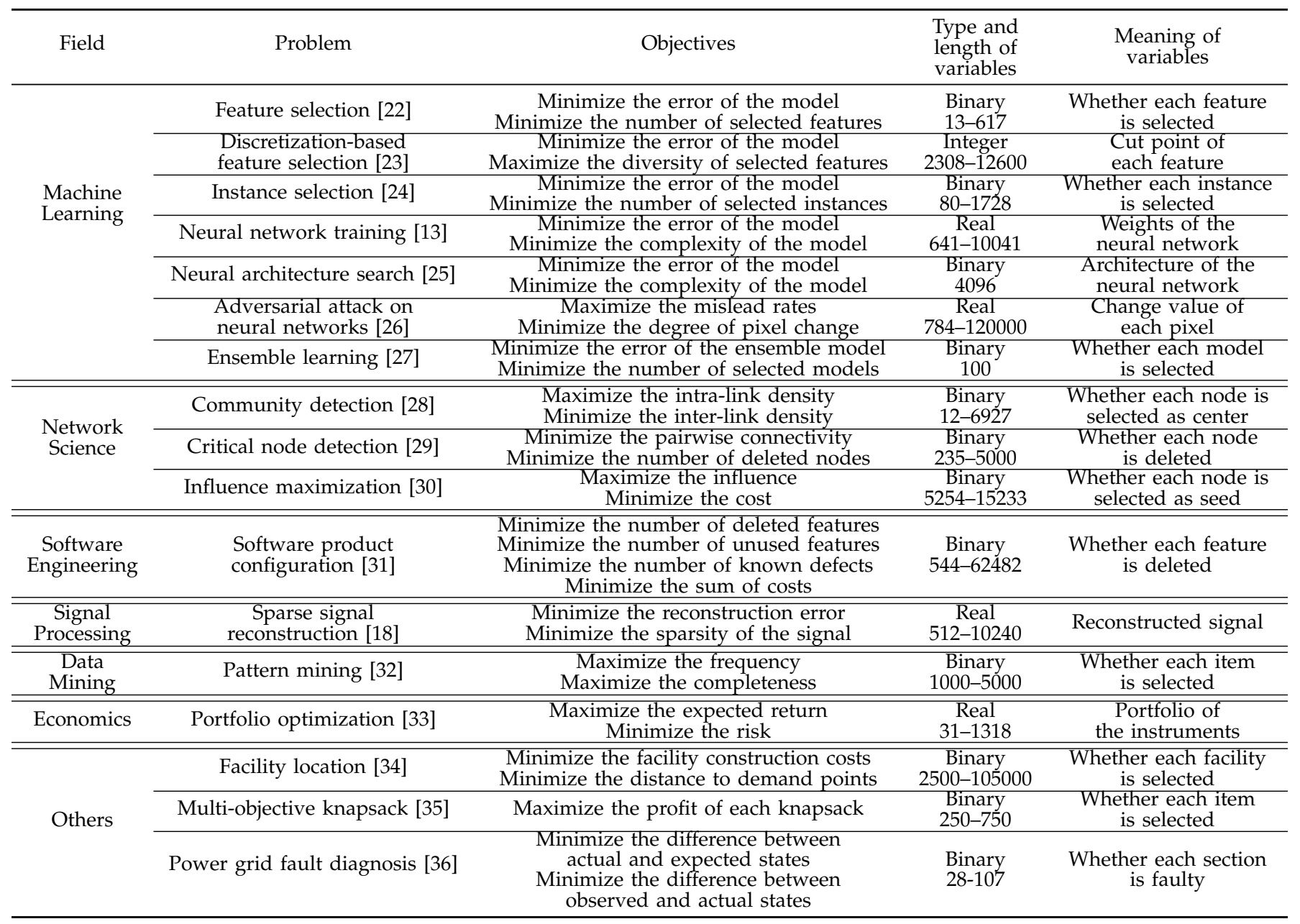

where the number of community centers is much less than the number of nodes; the power grid fault diagnosis problem [39] aims to detect all the faulty sections, which are usually much less than the normal sections.

Although the above problems have been tackled by some MOEAs as listed in Table I, these MOEAs are restricted to the objective functions and data structure of a specific problem, which cannot be used to solve different sparse LMOPs in a black-box manner. Therefore, it is necessary to develop a generic MOEAs for solving sparse LMOPs. Besides, existing large-scale MOEAs are inefficient for solving sparse LMOPs due to the computationally expensive objectives. For example, large-scale MOEAs can well solve an LMOP with 5,000 decision variables by using 200,000,000 function evaluations [8], but it is impractical to train a deep neural network for so many times for solving the neural architecture search problem [25]. Considering the sparse nature of Pareto optimal solutions, the Pareto optimal subspace can be approximated by ignoring the dimensions where the decision variables in Pareto optimal solutions are zero. Then, MOEAs can search for better solutions in the learnt subspace instead of the original search space. In other words, the original search space is drastically reduced.

Following this idea, this paper proposes a Pareto optimal subspace learning based MOEA for solving sparse LMOPs, which will be applied to eight real-world problems selected from Table I. In the next subsection, the Pareto optimal subspace learning approaches in existing MOEAs are reviewed.

\section{B. Existing Pareto Optimal Subspace Learning Approaches}

The Pareto optimal subspace learning approaches in existing MOEAs are mainly based on classical machine learning techniques. In [40], two genetic algorithms were proposed for solving MOPs with low effective dimensions. The low effective dimensions indicate that the objective functions are only related to a small proportion of the decision variables, hence the Pareto optimal subspace can be easily learnt by ignoring many useless dimensions. The algorithms in [40] adopt random embedding to ignore decision variables randomly, which is theoretically verified to keep the Pareto optimal subspace unchanged. On the other hand, some MOEAs adopt principal component analysis (PCA) to learn the Pareto optimal subspace directly. Based on the condition that the 
Pareto optimal solutions lie on an $(M-1)$-dimensional manifold [14], a regularity model-based multi-objective estimation of distribution algorithm was proposed in [15]. This MOEA uses an $(M-1)$-dimensional local PCA to partition the population into several clusters, and generates offspring solutions around each cluster centroid. Sun et al. [41] suggested a two-stage MOEA with Pareto optimal subspace learning, called MaOEAIT. In the first stage, the algorithm searches for some well-converged solutions by considering only the population convergence. In the second stage, it uses these solutions to learn the Pareto optimal subspace via PCA, and generates offspring solutions in the learnt subspace for enhancing the population diversity.

Although the idea of Pareto optimal subspace learning has been successfully adopted in a few MOEAs, they are not suited for solving sparse LMOPs due to the following reasons. Firstly, these approaches learn the Pareto optimal subspace by linearly reducing the decision variables, whereas the interactions between the decision variables of many real-world sparse LMOPs are nonlinear. Secondly, these approaches are tailored for specific types of optimization problems, which do not consider the sparse nature of LMOPs. Thirdly, these approaches can only handle continuous decision variables, which are unsuitable for many sparse LMOPs with binary variables.

As reported in [42], a hybrid representation of solution is effective for solving sparse LMOPs, where each solution is represented by a binary vector denoting the mask and a real vector denoting the decision variables. Following this idea, the proposed MOEA/PSL adopts RBM and DAE to learn the Pareto optimal subspace from the solutions with hybrid representation. More specifically, RBM is adopted to learn a sparse distribution from the binary vectors due to its ability of learning the probability distribution of the input obeying a binomial distribution, and DAE is adopted to learn a compact representation from the real vectors due to its ability of learning the compact representation of the input in continuous space. By adopting both RBM and DAE, the proposed MOEA/PSL can control the sparsity of solutions and learn a low-dimensional Pareto optimal subspace, thus highly improving the efficiency in finding sparse and well-converged solutions. In the experiments in Section IV-E, the utilization of both RBM and DAE will be verified by comparing them with a single RBM, $\mathrm{DAE}$, and some other techniques.

In the next subsection, some basic concepts about RBM and DAE are introduced.

\section{Restricted Boltzmann Machine (RBM) and Denoising Autoencoder (DAE)}

RBM [20] is the building block of deep belief network, which can reduce the dimensionality of input data in an unsupervised manner. As can be seen from Fig. 1(a), a typical RBM consists of an input layer and a hidden

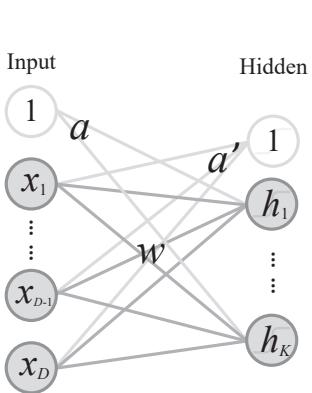

(a) Restricted Boltzmann machine

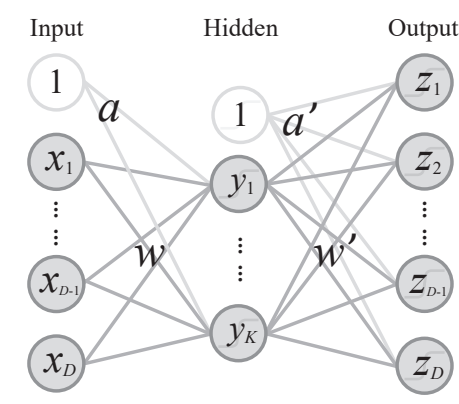

(b) Autoencoder
Fig. 1. General structures of restricted Boltzmann machine and autoencoder.

layer, where the nodes in the two layers are binary variables obeying a binomial distribution [43]. Given a vector $\mathbf{x}$ as the input, the value of each node $h_{j}$ in the hidden layer is set to 1 with a probability

$$
p\left(h_{j}=1 \mid \mathbf{x}\right)=\sigma\left(a_{j}+\Sigma_{i} x_{i} w_{i j}\right),
$$

where $a_{j}$ is the bias, $w_{i j}$ is the weight, and $\sigma(x)=$ $1 /(1+\exp (-x))$ is the sigmoid function. In practice, the value of $h_{j}$ is sampled by comparing $p\left(h_{j}=1 \mid \mathbf{x}\right)$ with a uniformly distributed random value within $[0,1]$. Similarly, the reconstructed value of each node $x_{i}$ in the input layer is set to 1 with a probability

$$
p\left(x_{i}^{\prime}=1 \mid \mathbf{h}\right)=\sigma\left(a_{j}^{\prime}+\Sigma_{j} h_{j} w_{i j}\right) .
$$

The goal of training RBM is to minimize the reconstruction error (i.e., the difference between the reconstructed vector $\mathrm{x}^{\prime}$ and the original input $\mathrm{x}$ ) by finding the optimal values of $\mathbf{a}, \mathbf{a}^{\prime}$ and $\mathbf{w}$, which can be achieved by the contrastive divergence algorithm [44].

Autoencoder (AE) [45] is the building block of stacked $\mathrm{AE}$, which is also used for dimensionality reduction. Different from RBM, AE is a three-layer network working in continuous space. As shown in Fig. 1(b), the value of each node $y_{j}$ in the hidden layer can be calculated by

$$
y_{j}=\sigma\left(a_{j}+\Sigma_{i} x_{i} w_{i j}\right),
$$

and the value of each node $z_{i}$ in the output layer can be calculated by

$$
z_{i}=\sigma\left(a_{i}^{\prime}+\Sigma_{j} y_{j} w_{j i}^{\prime}\right) .
$$

The reconstruction error of $\mathrm{AE}$ is the difference between the output $\mathrm{z}$ and the input $\mathrm{x}$, hence $\mathrm{AE}$ can be trained by the same way as feedforward neural network [46]. As for $\mathrm{DAE}$ [21], it is a popular variant of AE that enforces the robustness by adding noise to the input. In this work, each input $\mathrm{x}$ for DAE is perturbed by randomly setting the elements of $\mathrm{x}$ to zero.

Although the above neural networks have been adopted in estimation of distribution algorithm [47] and evolutionary multitasking algorithm [48], they have not been used in Pareto optimal subspace learning for solving LMOPs before. In this work, RBM and DAE are 


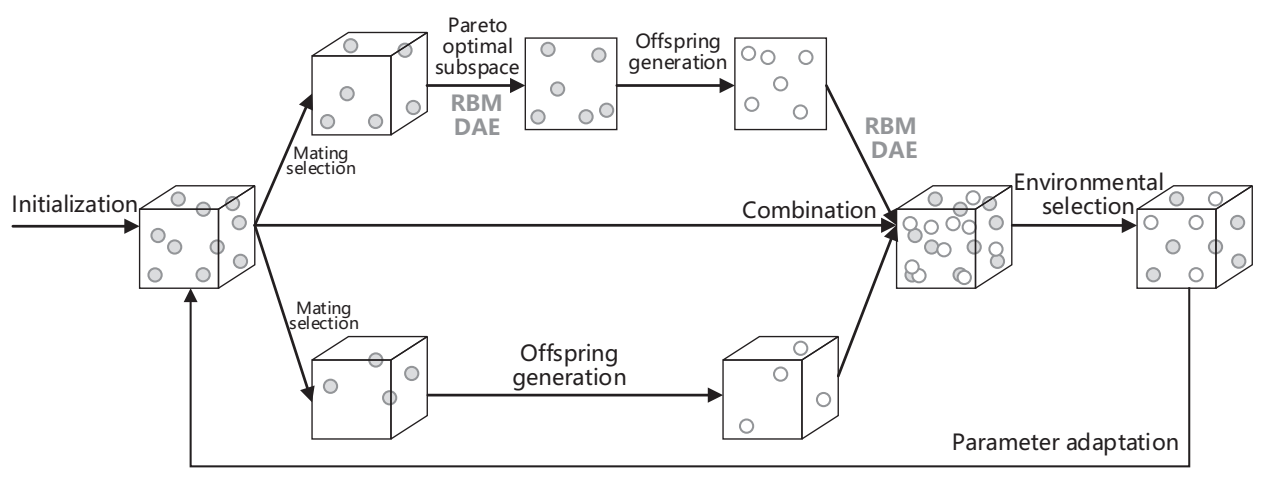

Fig. 2. Procedure of MOEA/PSL.

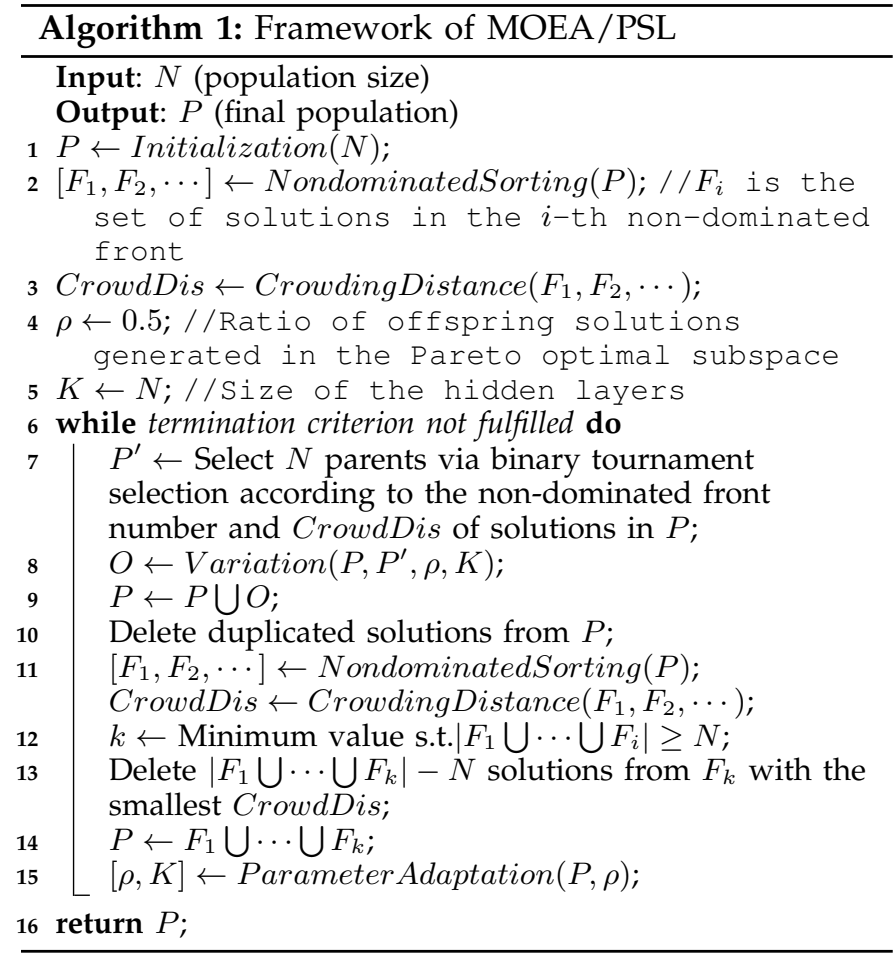

adopted to learn a sparse distribution and a compact representation of the decision variables, respectively, where the combination of the learnt sparse distribution and compact representation is regarded as an approximation of the Pareto optimal subspace. In the next section, the procedure of the proposed algorithm is elaborated.

\section{The Proposed Algorithm}

\section{A. Framework of MOEA/PSL}

The general framework of MOEA/PSL is presented in Fig. 2 and Algorithm 1. To begin with, $N$ solutions are initialized by the Latin hypercube sampling method [49] to form the initial population $P$, and the non-dominated front number [50] and crowding distance [6] of each solution are calculated. In the main loop, $N$ parents are selected by binary tournament selection and used to generate $N$ offspring solutions. The offspring set $O$ are then combined with the population $P$, and $N$ solutions with better non-dominated front numbers and crowding distances in $P \cup O$ will survive to the next generation. Finally, the parameters $\rho$ and $K$ are adapted according to the new population. As a consequence, the mating selection and environmental selection of MOEA/PSL are the same to those of NSGA-II [6], while MOEA/PSL generates some offspring solutions in a learnt subspace.

In the following two subsections, the core components of MOEA/PSL are described in detail.

\section{B. Pareto Optimal Subspace Learning and Offspring Gener- ation in MOEA/PSL}

The non-dominated solutions in the population are adopted as an approximation of the Pareto optimal solutions for learning the Pareto optimal subspace. To enable RBM and DAE to learn the sparse distribution and compact representation, respectively, each solution $\mathrm{x}$ is represented by a binary vector $\mathrm{xb}$ and a real vector $\mathrm{xr}$, and each decision variable of $\mathrm{x}$ is obtained by

$$
x_{i}=x b_{i} \times x r_{i},
$$

where $x b_{i}$ indicates whether the $i$-th decision variable is zero, and $x r_{i}$ indicates the real value of the $i$-th decision variable. If the problem is with binary variables, the real vector $\mathrm{xr}$ is always set to a vector of ones. In short, each solution is represented by $\mathbf{x b}$ and $\mathbf{x r}$ instead of $\mathbf{x}$, where the length of all the three vectors equals to the number of decision variables. Before generating offspring solutions, the binary vectors of all the non-dominated solutions are used to train an RBM via the contrastive divergence algorithm, and the real vectors of all the non-dominated solutions are used to train a DAE via gradient descent. As shown in Fig. 3, the real vector and binary vector of each solution can be reduced by the representation of the hidden layers of the two neural networks, and the reduced vectors can also be recovered to normal vectors. In other words, each solution can be mapped between the original search space and the Pareto optimal subspace, where offspring solutions are generated in the Pareto optimal subspace and evaluated by the objective functions in the original search space.

Afterwards, two parents are randomly picked up from the mating pool each time, whose binary vectors are 


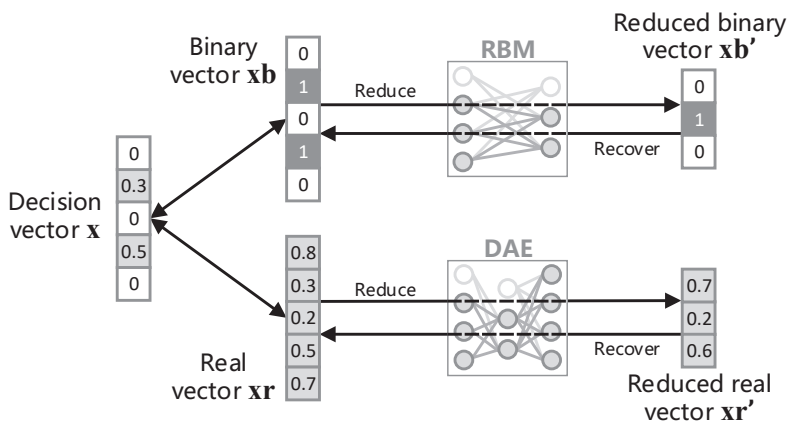

Fig. 3. Reduction and recover of solutions in MOEA/PSL. Note that the variables $\mathbf{x b}$ and $\mathbf{x r}$ are stored in each solution, while $\mathbf{x}$ is a temporary variable for calculating objective functions.

used to generate the binary vectors of two offspring solutions by single-point crossover and bitwise mutation, and real vectors are used to generate the real vectors of two offspring solutions by simulated binary crossover [51] and polynomial mutation [52]. It is worth to note that a parameter $\rho$ is used to determine whether each offspring solution is generated in the Pareto optimal subspace or the original search space. Specifically, the parameter $\rho$ is compared with a random value within $[0,1]$. If $\rho$ is larger than the random value, the binary vectors and real vectors of the parents are reduced by (1) and (3), respectively, and the offspring solutions are generated in the Pareto optimal subspace and then recovered by (2) and (4); otherwise, the offspring solutions are generated in the original search space without using RBM or DAE. Algorithm 2 summarizes the procedure of the offspring generation strategy.

\section{Parameter Adaptation Strategy in MOEA/PSL}

There are two parameters related to the offspring generation in MOEA/PSL, i.e., the ratio of offspring solutions generated in the Pareto optimal subspace $\rho$ and the size of the hidden layers $K$. Intuitively, the parameter $\rho$ should be dynamically adjusted to better balance exploration and exploitation, and the parameter $K$ should decrease with the convergence of the population. To this end, a parameter adaptation strategy is designed to automatically determine the values of $\rho$ and $K$ at each generation. Specifically, the parameter $\rho$ is iteratively updated by

$$
\rho_{t+1}=0.5 \times\left(\rho_{t}+\frac{s_{1, t}}{s_{1, t}+s_{2, t}}\right),
$$

where $\rho_{t}$ denotes the value of $\rho$ at the $t$-th generation and $\rho_{0}=0.5, s_{1, t}$ denotes the number of successful offspring solutions generated in the Pareto optimal subspace at the $t$-th generation, and $s_{2, t}$ denotes the number of successful offspring solutions generated in the original search space at the $t$-th generation. A successful offspring solution means that it survives to the next generation, hence the ratio $\frac{s_{1, t}}{s_{1, t}+s_{2, t}}$ reflects the effectiveness of generating offspring solutions in the Pareto optimal subspace. In short, the parameter $\rho$ is updated according to the

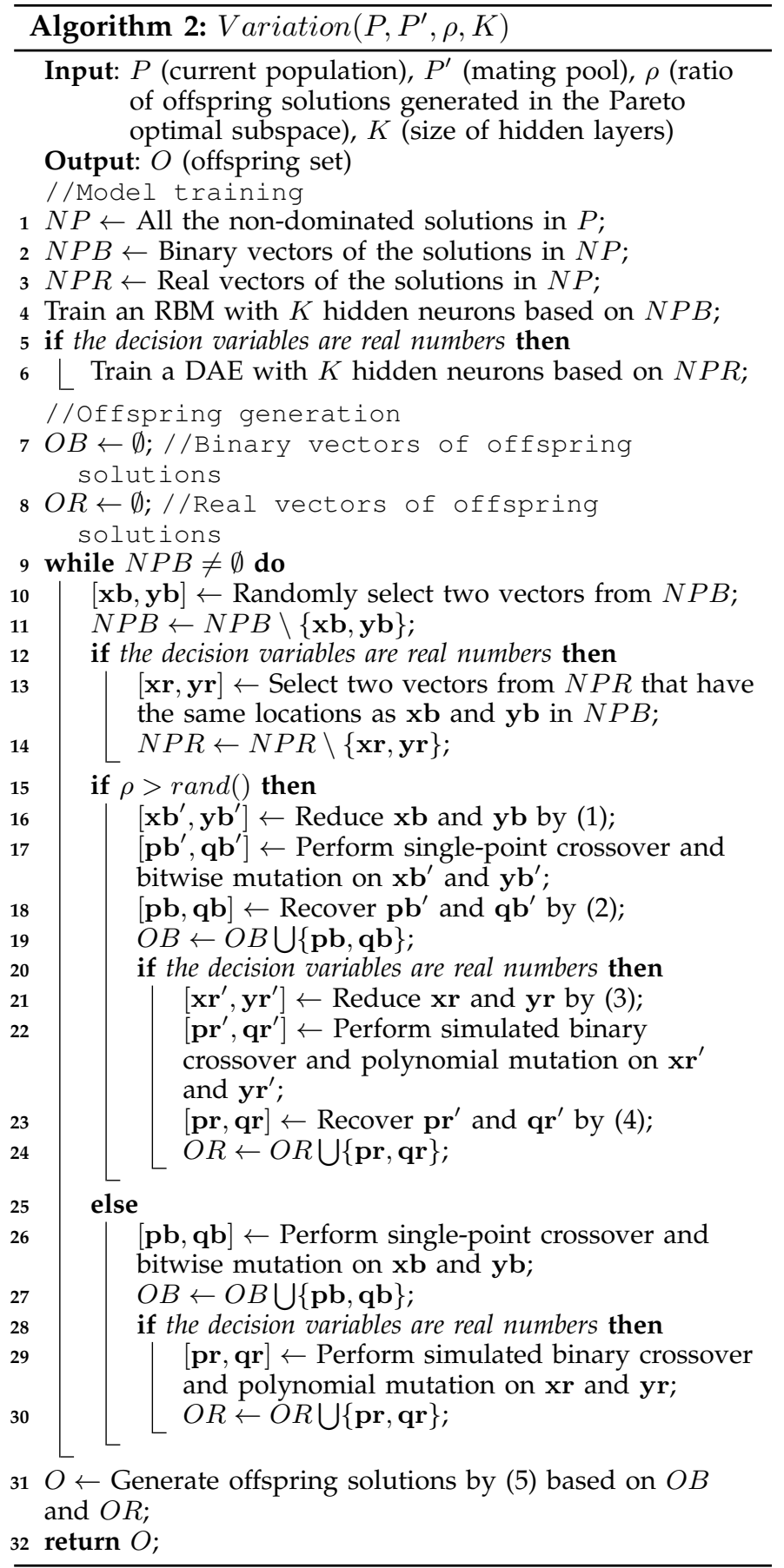

number of successful offspring solutions generated at the previous generations, where a higher ratio of successful offspring solutions generated in the Pareto optimal subspace results in a larger $\rho$, and vice versa.

By contrast, the parameter $K$ is determined according to the sparsity of the non-dominated solutions in the current population. Let dec be a binary vector denoting whether each decision variable should be nonzero, the probability that $d e c_{i}$ is set to 1 is estimated according to the non-dominated solution set $N P$ :

$$
p\left(\operatorname{dec}_{i}=1 \mid N P\right)=\frac{1}{|N P|} \sum_{\mathbf{x} \in N P}\left|\operatorname{sign}\left(x_{i}\right)\right|,
$$




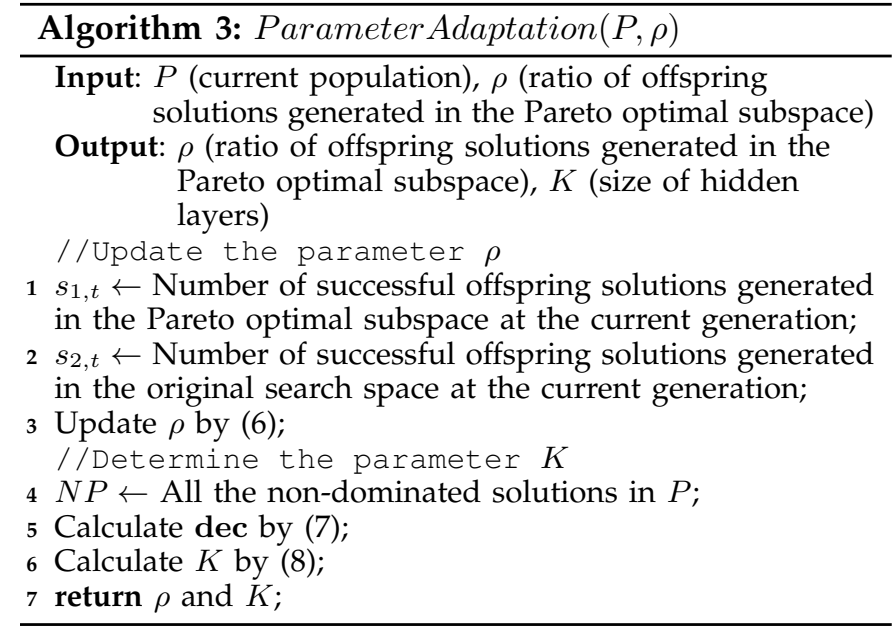

where $\left|\operatorname{sign}\left(x_{i}\right)\right|$ equals to 0 if $x_{i}=0$ and 1 otherwise. Then, the value of $d e c_{i}$ is sampled by comparing the probability with a uniformly distributed random value within $[0,1]$. Since the zero elements in dec (i.e., the sparse part of the decision variables) can be ignored, the size of the hidden layers is set to the number of nonzero elements in dec, i.e.,

$$
K=\sum \operatorname{dec}_{i}
$$

The pseudocode of the parameter adaptation strategy in MOEA/PSL is given in Algorithm 3.

\section{Computational Complexity of MOEA/PSL}

Each generation of MOEA/PSL consists of four main steps, i.e., Pareto optimal subspace learning, offspring generation, environmental selection, and parameter adaptation. The time complexity of training neural networks in Pareto optimal subspace learning is $O(N E D K)$, where $N, E, D$, and $K$ denotes the population size, the number of epochs for training, the number of decision variables, and the hidden layer size, respectively. The time complexity of generating offspring solutions is $O(N D K)$. The environmental selection is the same to that of NSGA-II, which has a time complexity of $O\left(M N^{2}\right)$ [6], where $M$ denotes the number of objectives. The time complexity of parameter adaptation is $O(N D)$. To summarize, the total computational complexity of MOEA/PSL in one generation is $O\left(M N^{2}+N E D K\right)$.

\section{EMPIRICAL STUDIES}

To empirically investigate the performance of MOEA/PSL, it is first compared with four representative MOEAs on eight benchmark problems with sparse Pareto optimal solutions. Then, MOEA/PSL is tested on eight real-world problems from various fields. Afterwards, the effectiveness of the two neural networks in Pareto optimal subspace learning is verified. Finally, the effectiveness of the parameter adaptation strategy in MOEA/PSL is verified. All the experiments are conducted on PlatEMO [53].

\section{A. Comparative Algorithms}

Four state-of-the-art MOEAs are selected as baselines in the experiments, namely, LMEA [8], WOF-SMPSO [11], MaOEA-IT [41], and SparseEA [42]. LMEA is a divide-and-conquer MOEA tailored for LMOPs, which divides the decision variables into convergence-related variables and diversity-related variables and optimizes them separately. WOF-SMPSO refers to the WOF based speed-constrained multi-objective particle swarm optimization (PSO) algorithm, which is efficient for LMOPs due to the fast convergence speed of PSO and a problem transformation strategy. MaOEA-IT uses PCA to learn the Pareto optimal subspace according to a set of wellconverged solutions. Besides, SparseEA is currently the only MOEA considering the sparse nature of problems, but it encounters difficulties when dealing with a large number of decision variables due to the absence of Pareto optimal subspace learning.

Population size and number of function evaluations. The population size and number of function evaluations of all the compared MOEAs are set to the same for fair comparisons. For benchmark problems, the population size is set to 100 and the number of function evaluations is set to $100 \times D$, where $D$ denotes the number of decision variables. Due to the computationally expensive objectives of real-world problems, the population size is set to 50 as suggested in [42]; besides, the number of function evaluations is set to $2.0 \times 10^{4}, 1.0 \times 10^{5}$ and $2.0 \times 10^{5}$ for problems with approximately 1000,5000 and 10000 real variables, and set to $1.0 \times 10^{4}, 5.0 \times 10^{4}$ and $1.0 \times 10^{5}$ for problems with approximately 1000,5000 and 10000 binary variables.

Genetic operators. In LMEA, MaOEA-IT, SparseEA, and MOEA/PSL, the single-point crossover and bitwise mutation are employed for solving problems with binary variables, and the simulated binary crossover [51] and polynomial mutation [52] are employed for solving problems with real variables; the probability of crossover is set to 1 , the probability of mutation is set to $1 / D$, and the distribution index of both crossover and mutation is set to 20. In WOF-SMPSO, the PSO operator [57] and polynomial mutation are employed for solving all the problems; when handling binary variables, it optimizes the same number of real variables within $[0,1]$ and rounds the variables before calculating objective values.

Other parameters. The other parameters in all the MOEAs are tuned for a relatively good performance. For LMEA, the number of selected solutions for variable clustering is set to 2, the number of perturbations on each solution for variable clustering is set to 4 , and the number of selected solutions for variable interaction analysis is set to 5. For WOF-SMPSO, the number of groups is set to 4 , the number of evaluations for original problem is set to 1000 , the number of evaluations for transformed problem is set to 500, the number of chosen solutions for weight optimization is set to 3 , and the fraction of evaluations for weight optimization is set 
TABLE II

DATASETS OF EIGHT SPARSE LMOPS IN REAL-WORLD APPLICATIONS.

\begin{tabular}{|c|c|c|c|c|c|c|}
\hline $\begin{array}{c}\text { Feature selection } \\
\text { problem }\end{array}$ & $\begin{array}{c}\text { Type of } \\
\text { variables }\end{array}$ & $\begin{array}{c}\text { No. of } \\
\text { variables }\end{array}$ & Dataset & No. of samples & No. of features & No. of classes \\
\hline FS1 & \multirow{3}{*}{ Binary } & 800 & Gse72526 & 61 & 800 & 4 \\
\hline FS2 & & 5966 & Prostate $\mathrm{GE}^{2}$ & 102 & 5966 & 2 \\
\hline FS3 & & 10000 & Arcene $^{2}$ & 200 & 10000 & 2 \\
\hline $\begin{array}{l}\text { Instance selection } \\
\text { problem }\end{array}$ & $\begin{array}{c}\text { Type of } \\
\text { variables }\end{array}$ & $\begin{array}{c}\text { No. of } \\
\text { variables }\end{array}$ & Dataset & No. of samples & No. of features & No. of classes \\
\hline IS1 & \multirow{3}{*}{ Binary } & 862 & Fouclass $^{3}$ & 862 & 3 & 2 \\
\hline IS2 & & 4177 & Abalone ${ }^{4}$ & 4177 & 9 & 2 \\
\hline IS3 & & 11055 & phishing $^{3}$ & 11055 & 68 & 2 \\
\hline $\begin{array}{l}\text { Neural network } \\
\text { training problem }\end{array}$ & $\begin{array}{c}\text { Type of } \\
\text { variables }\end{array}$ & $\begin{array}{c}\text { No. of } \\
\text { variables }\end{array}$ & Dataset & No. of samples & No. of features & No. of classes \\
\hline NN1 & \multirow{3}{*}{ Real } & 1181 & Spambase $^{4}$ & 4597 & 57 & 2 \\
\hline NN2 & & 5161 & Semeion Handwritten Digit ${ }^{4}$ & 1593 & 256 & 10 \\
\hline NN3 & & 10041 & Madelon ${ }^{4}$ & 2600 & 500 & 2 \\
\hline $\begin{array}{l}\text { Community detection } \\
\text { problem }\end{array}$ & $\begin{array}{c}\text { Type of } \\
\text { variables }\end{array}$ & $\begin{array}{c}\text { No. of } \\
\text { variables }\end{array}$ & Dataset & No. of nodes & No. of edges & \\
\hline CD1 & \multirow{3}{*}{ Binary } & 1133 & Email $^{5}$ & 1133 & 5451 & \\
\hline CD2 & & 4039 & Facebook [54] & 4039 & 88234 & \\
\hline CD3 & & 9885 & Duke14 [54] & 9885 & 506437 & \\
\hline $\begin{array}{c}\text { Critical node } \\
\text { detection problem }\end{array}$ & $\begin{array}{l}\text { Type of } \\
\text { variables }\end{array}$ & $\begin{array}{c}\text { No. of } \\
\text { variables }\end{array}$ & Dataset & No. of nodes & No. of edges & \\
\hline $\begin{array}{l}\text { CN1 } \\
\text { CN2 } \\
\text { CN3 }\end{array}$ & Binary & $\begin{array}{l}1176 \\
4039 \\
9885\end{array}$ & $\begin{array}{l}\text { Power Network [54] } \\
\text { Facebook [54] } \\
\text { Duke14 [54] }\end{array}$ & $\begin{array}{l}1176 \\
4039 \\
9885\end{array}$ & $\begin{array}{c}8688 \\
88234 \\
506437\end{array}$ & \\
\hline $\begin{array}{c}\text { Sparse signal } \\
\text { reconstruction problem }\end{array}$ & $\begin{array}{c}\text { Type of } \\
\text { variables }\end{array}$ & $\begin{array}{c}\text { No. of } \\
\text { variables }\end{array}$ & Dataset & Length of signal & $\begin{array}{c}\text { Length of received } \\
\text { signal }\end{array}$ & Sparsity of signal \\
\hline SR1 & \multirow{3}{*}{ Real } & 1024 & Synthetic [55] & 1024 & 480 & 260 \\
\hline SR2 & & 5120 & Synthetic [55] & 5120 & 2400 & 1300 \\
\hline SR3 & & 10240 & Synthetic [55] & 10240 & 4800 & 2600 \\
\hline $\begin{array}{c}\text { Pattern mining } \\
\text { problem }\end{array}$ & $\begin{array}{c}\text { Type of } \\
\text { variables }\end{array}$ & $\begin{array}{c}\text { No. of } \\
\text { variables }\end{array}$ & Dataset & No. of transactions & No. of items & $\begin{array}{l}\text { Avg. length of } \\
\text { transactions }\end{array}$ \\
\hline PM1 & \multirow{3}{*}{ Binary } & 1000 & Synthetic [56] & 10000 & 1000 & 500 \\
\hline PM2 & & 5000 & Synthetic [56] & 50000 & 5000 & 2500 \\
\hline PM3 & & 10000 & Synthetic [56] & 100000 & 10000 & 5000 \\
\hline $\begin{array}{c}\text { Portfolio } \\
\text { optimization problem }\end{array}$ & $\begin{array}{c}\text { Type of } \\
\text { variables }\end{array}$ & $\begin{array}{c}\text { No. of } \\
\text { variables }\end{array}$ & Dataset & No. of instruments & $\begin{array}{l}\text { Length of each } \\
\text { instrument }\end{array}$ & \\
\hline PO1 & \multirow{3}{*}{ Real } & 1000 & EURCHF $^{6}$ & 1000 & 50 & \\
\hline $\mathrm{PO} 2$ & & 5000 & EURCHF $^{6}$ & 5000 & 50 & \\
\hline PO3 & & 10000 & EURCHF $^{6}$ & 10000 & 50 & \\
\hline
\end{tabular}

1. https://www.ncbi.nlm.nih.gov/geo/query/acc.cgi 2. http://featureselection.asu.edu/datasets.php

3. https://www.csie.ntu.edu.tw/\%7ecjlin/libsvmtools/datasets/binary.html 4. https://archive.ics.uci.edu/ml

5. http://deim.urv.cat/\%7ealexandre.arenas/data/welcome.htm 6. https://www.metatrader5.com/en

to 0.5. For MaOEA-IT, the number of evaluations for dynamic weight aggregation is set to $50 \times D$, and the number of evaluations for reference lines mapping is set to $D$. For MOEA/PSL, the number of epochs for training neural networks is set to 10 .

\section{B. Test Problems}

The sparse multi-objective test suite [42] is adopted to test the performance of the compared MOEAs, which contains eight benchmark problems SMOP1-SMOP8 with scalable number of decision variables. These problems are characterized by multi-modality, deception, epistasis, and low intrinsic dimensionality, posing various difficulties for MOEAs to obtain a set of sparse solutions. In the experiments, the number of objectives of these problems is set to 2, the number of decision variables is set to 1000, 5000 and 10000, and the sparsity of Pareto optimal solutions is set to 0.1.

Moreover, eight sparse LMOPs in real-world applications are established as test problems, including feature selection, instance selection, neural network training, community detection, critical node detection, sparse signal reconstruction, pattern mining, and portfolio optimization. The mathematical definitions of these applications can be found in Supplementary Materials I.
As shown in Table II, three datasets are used in each application, which result in three sparse LMOPs with approximately 1000, 5000 and 10000 decision variables.

For each MOEA on each test problem, 30 independent runs are performed to obtain statistical results, where the IGD indicator [58] with 10000 reference points is used to measure the results on benchmark problems. Since the Pareto fronts of real-world problems are unknown, the HV indicator [59] with a reference point $(1,1)$ is used to measure the results on real-world problems. Besides, the Wilcoxon rank sum test with a significance level of 0.05 is adopted to perform statistical analysis, where the symbols ' + ', ' - ' and ' $\approx$ ' indicate that the result by another MOEA is significantly better, significantly worse, and statistically similar to that obtained by the proposed MOEA/PSL, respectively.

\section{Results on Benchmark Problems}

Table III lists the IGD values obtained by the five compared MOEAs on SMOP1-SMOP8 with 1000, 5000 and 10000 decision variables. In general, the proposed MOEA/PSL performs the best on 19 out of 24 test instances, SparseEA performs the best on 5 test instances, while LMEA, WOF-SMPSO, and MaOEA-IT do not obtain any best result. As a consequence, the experimental 
TABLE III

IGD VAlues ObTAINed by LMEA, WOF-SMPSO, MAOEA-IT, SPARSEEA, AND THE PROPOSEd MOEA/PSL ON SMOP1-SMOP8, WHERE the Best Result in Each RoW is Highlighted.

\begin{tabular}{|c|c|c|c|c|c|c|}
\hline Problem & Dec & LMEA & WOF-SMPSO & MaOEA-IT & SparseEA & MOEA/PSL \\
\hline \multirow{3}{*}{ SMOP1 } & 1000 & $8.2758 \mathrm{e}-1(8.25 \mathrm{e}-3)-$ & $2.3787 \mathrm{e}-1(2.38 \mathrm{e}-2)-$ & $4.7235 e-1(7.75 e-2)-$ & $2.8467 \mathrm{e}-2(2.12 \mathrm{e}-17)-$ & $1.1507 \mathrm{e}-2(2.11 \mathrm{e}-3)$ \\
\hline & 5000 & $8.6219 \mathrm{e}-1(3.48 \mathrm{e}-3)-$ & $1.7494 \mathrm{e}-1(2.52 \mathrm{e}-2)-$ & 7.5928e-1 (3.41e-2) - & $3.8535 \mathrm{e}-2(1.25 \mathrm{e}-3)-$ & $1.3438 e-2(2.33 e-3)$ \\
\hline & 10000 & $8.7141 \mathrm{e}-1(1.98 \mathrm{e}-3)-$ & $1.4972 \mathrm{e}-1(1.56 \mathrm{e}-2)-$ & $9.3680 \mathrm{e}-1(5.54 \mathrm{e}-2)-$ & $4.0795 \mathrm{e}-2(7.77 \mathrm{e}-4)-$ & $1.4308 \mathrm{e}-2(4.31 \mathrm{e}-3)$ \\
\hline \multirow{3}{*}{ SMOP2 } & 1000 & $1.7590 \mathrm{e}+0(7.28 \mathrm{e}-3)-$ & $3.2865 \mathrm{e}-1(1.46 \mathrm{e}-1)-$ & $1.1721 \mathrm{e}+0(5.36 \mathrm{e}-2)-$ & $6.4140 \mathrm{e}-2(4.23 \mathrm{e}-17)-$ & $4.2070 \mathrm{e}-2(9.70 \mathrm{e}-3)$ \\
\hline & 5000 & $1.7838 e+0(3.03 e-3)-$ & $1.8621 \mathrm{e}-1(2.67 \mathrm{e}-2)-$ & $1.6124 \mathrm{e}+0(3.05 \mathrm{e}-2)-$ & $9.9443 e-2(2.23 e-3)-$ & $4.7483 e-2(7.87 e-3)$ \\
\hline & 10000 & $1.7912 \mathrm{e}+0(1.56 \mathrm{e}-3)-$ & $1.8004 \mathrm{e}-1(1.13 \mathrm{e}-2)-$ & $1.7619 \mathrm{e}+0(3.55 \mathrm{e}-2)-$ & $1.0450 \mathrm{e}-1(3.13 \mathrm{e}-3)-$ & $5.5203 e-2(3.48 e-3)$ \\
\hline \multirow{3}{*}{ SMOP3 } & 1000 & $2.1679 \mathrm{e}+0(1.02 \mathrm{e}-2)-$ & $7.0334 \mathrm{e}-1(2.76 \mathrm{e}-3)-$ & $1.5729 \mathrm{e}+0(5.55 \mathrm{e}-2)-$ & $3.2951 \mathrm{e}-2(2.12 \mathrm{e}-17)-$ & $1.2598 \mathrm{e}-2(3.06 \mathrm{e}-3)$ \\
\hline & 5000 & $2.1960 \mathrm{e}+0(4.31 \mathrm{e}-3)-$ & 7.0172e-1 (1.12e-3) - & $2.0555 \mathrm{e}+0(3.81 \mathrm{e}-2)-$ & $4.2581 \mathrm{e}-2(1.40 \mathrm{e}-3)-$ & $1.2200 \mathrm{e}-2(8.68 \mathrm{e}-4)$ \\
\hline & 10000 & $2.2020 \mathrm{e}+0(2.46 \mathrm{e}-3)-$ & 7.0123e-1 (6.11e-4) - & $2.1973 \mathrm{e}+0(1.82 \mathrm{e}-2)-$ & $4.5652 \mathrm{e}-2(6.62 \mathrm{e}-4)-$ & $1.5615 \mathrm{e}-2(7.36 \mathrm{e}-4)$ \\
\hline \multirow{3}{*}{ SMOP4 } & 1000 & $8.8105 \mathrm{e}-1(3.87 \mathrm{e}-3)-$ & $6.0319 \mathrm{e}-2(7.03 \mathrm{e}-2)-$ & $5.9578 \mathrm{e}-1(5.38 \mathrm{e}-2)-$ & $4.5184 \mathrm{e}-3(8.82 \mathrm{e}-19)+$ & $4.7175 e-3(2.47 e-4)$ \\
\hline & 5000 & $8.9139 \mathrm{e}-1(1.81 \mathrm{e}-3)-$ & $9.4086 \mathrm{e}-3(4.94 \mathrm{e}-3)-$ & $8.0334 \mathrm{e}-1(1.80 \mathrm{e}-2)-$ & $4.7526 \mathrm{e}-3(2.36 \mathrm{e}-4) \approx$ & $4.7211 \mathrm{e}-3(1.14 \mathrm{e}-4)$ \\
\hline & 10000 & $8.9378 \mathrm{e}-1(1.75 \mathrm{e}-3)-$ & $1.0315 \mathrm{e}-2(1.04 \mathrm{e}-2)-$ & $8.6391 \mathrm{e}-1(2.41 \mathrm{e}-2)-$ & $5.0535 \mathrm{e}-3(3.72 \mathrm{e}-4)-$ & $4.5711 \mathrm{e}-3(1.11 \mathrm{e}-4)$ \\
\hline \multirow{3}{*}{ SMOP5 } & 1000 & $6.0578 \mathrm{e}-1(5.70 \mathrm{e}-3)-$ & $3.5623 \mathrm{e}-1(1.80 \mathrm{e}-3)-$ & $4.4576 \mathrm{e}-1(1.72 \mathrm{e}-2)-$ & $5.8769 \mathrm{e}-3(2.84 \mathrm{e}-4)+$ & $7.4279 \mathrm{e}-3(4.60 \mathrm{e}-4)$ \\
\hline & 5000 & $6.2194 \mathrm{e}-1(3.01 \mathrm{e}-3)-$ & $3.4946 \mathrm{e}-1(5.59 \mathrm{e}-4)-$ & $5.4720 \mathrm{e}-1(1.43 \mathrm{e}-2)-$ & $5.8794 \mathrm{e}-3(2.35 \mathrm{e}-4)+$ & $6.8328 \mathrm{e}-3(2.47 \mathrm{e}-4)$ \\
\hline & 10000 & $6.2439 \mathrm{e}-1(1.40 \mathrm{e}-3)-$ & $3.4855 \mathrm{e}-1(2.53 \mathrm{e}-4)-$ & $6.2980 \mathrm{e}-1(1.63 \mathrm{e}-2)-$ & $6.0049 \mathrm{e}-3(1.39 \mathrm{e}-4)+$ & $6.6151 \mathrm{e}-3(1.72 \mathrm{e}-4)$ \\
\hline \multirow{3}{*}{ SMOP6 } & 1000 & $2.4791 \mathrm{e}-1(2.41 \mathrm{e}-3)-$ & $8.0357 \mathrm{e}-2(1.53 \mathrm{e}-3)-$ & $1.6209 \mathrm{e}-1(1.04 \mathrm{e}-2)-$ & $7.0559 \mathrm{e}-3(3.22 \mathrm{e}-4)+$ & $7.4349 \mathrm{e}-3(5.34 \mathrm{e}-4)$ \\
\hline & 5000 & $2.5654 \mathrm{e}-1(1.49 \mathrm{e}-3)-$ & $3.8829 \mathrm{e}-2(1.99 \mathrm{e}-2)-$ & $2.4433 e-1(8.07 e-3)-$ & 7.5168e-3 (2.35e-4) - & $6.9494 \mathrm{e}-3(3.12 \mathrm{e}-4)$ \\
\hline & 10000 & $2.5786 \mathrm{e}-1(7.80 \mathrm{e}-4)-$ & $2.0055 \mathrm{e}-2(1.07 \mathrm{e}-2)-$ & $2.9218 \mathrm{e}-1(8.71 \mathrm{e}-3)-$ & $7.5046 e-3(9.62 e-5)-$ & $6.8096 \mathrm{e}-3(3.51 \mathrm{e}-4)$ \\
\hline \multirow{3}{*}{ SMOP7 } & 1000 & $1.4609 \mathrm{e}+0(1.58 \mathrm{e}-2)-$ & $9.6058 \mathrm{e}-2(8.53 \mathrm{e}-3)-$ & $8.7400 e-1(8.53 e-2)-$ & $8.8565 \mathrm{e}-2(7.96 \mathrm{e}-3)-$ & $5.5240 \mathrm{e}-2(3.62 \mathrm{e}-2)$ \\
\hline & 5000 & $1.5238 \mathrm{e}+0(8.84 \mathrm{e}-3)-$ & $8.3179 \mathrm{e}-2(4.20 \mathrm{e}-3)-$ & $1.2876 \mathrm{e}+0(4.95 \mathrm{e}-2)-$ & $1.2660 \mathrm{e}-1(4.33 \mathrm{e}-3)-$ & $4.9372 \mathrm{e}-2(8.25 \mathrm{e}-3)$ \\
\hline & 10000 & $1.5406 \mathrm{e}+0(4.11 \mathrm{e}-3)-$ & $7.6538 \mathrm{e}-2(5.80 \mathrm{e}-3)-$ & $1.5120 e+0(9.26 e-2)-$ & $1.4665 \mathrm{e}-1(2.94 \mathrm{e}-2)-$ & $5.0672 \mathrm{e}-2(7.09 \mathrm{e}-3)$ \\
\hline \multirow{3}{*}{ SMOP8 } & 1000 & $3.2484 \mathrm{e}+0(1.31 \mathrm{e}-2)-$ & $5.6267 \mathrm{e}+0(2.23 \mathrm{e}-2)-$ & $2.4728 \mathrm{e}+0(6.67 \mathrm{e}-2)-$ & $2.4697 \mathrm{e}-1(1.49 \mathrm{e}-2)-$ & $2.2823 e-1(4.59 \mathrm{e}-2)$ \\
\hline & 5000 & $3.2995 e+0(4.10 e-3)-$ & $5.3577 \mathrm{e}-1(4.01 \mathrm{e}-3)-$ & $2.9442 \mathrm{e}+0(5.61 \mathrm{e}-2)-$ & $3.2577 \mathrm{e}-1(9.22 \mathrm{e}-3)-$ & $2.5555 e-1(3.72 e-2)$ \\
\hline & 10000 & $3.3093 \mathrm{e}+0(3.72 \mathrm{e}-3)-$ & $5.3185 \mathrm{e}-1(3.71 \mathrm{e}-3)-$ & $3.1224 \mathrm{e}+0(5.42 \mathrm{e}-2)-$ & $3.4426 \mathrm{e}-1(4.90 \mathrm{e}-3)-$ & $2.5127 \mathrm{e}-1(7.19 \mathrm{e}-3)$ \\
\hline
\end{tabular}

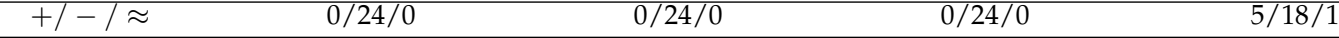

' + ' ' ' - ' and ' $\approx$ ' indicate that the result is significantly better, significantly worse, and statistically similar to that obtained by the proposed MOEA/PSL, respectively.
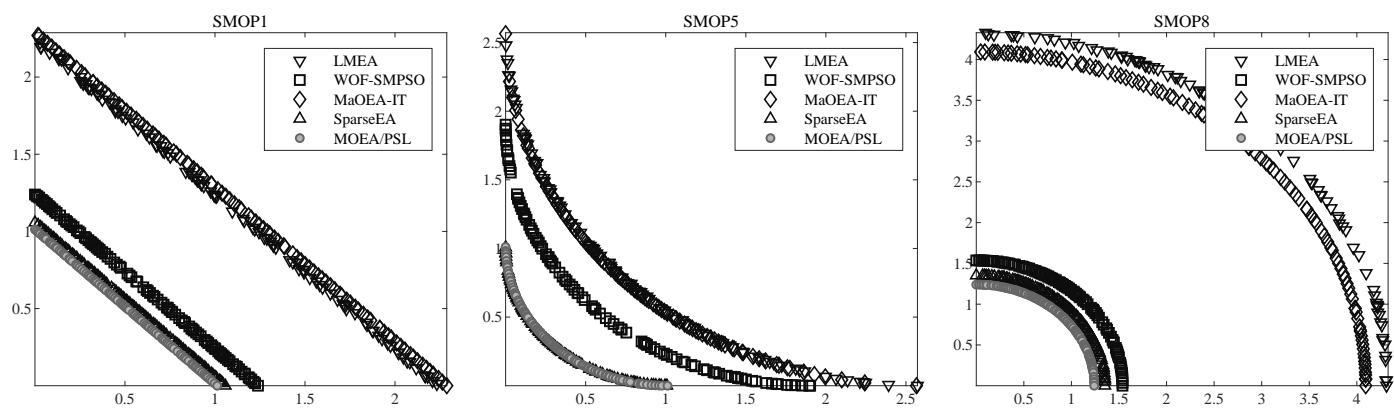

Fig. 4. Non-dominated solution sets with median IGD obtained by LMEA, WOF-SMPSO, MaOEA-IT, SparseEA, and the proposed MOEA/PSL on SMOP1, SMOP5, and SMOP8 with 10000 decision variables.

results of MOEA/PSL and SparseEA verify the importance of considering sparsity in solving sparse problems, and the superiority of MOEA/PSL over SparseEA verifies the effectiveness of Pareto optimal subspace learning in solving LMOPs.

For further observation, Fig. 4 plots the nondominated solution sets with median IGD values among 30 runs obtained by the five compared MOEAs on SMOP1, SMOP5, and SMOP8 with 10000 decision variables. For SMOP1 with a mostly unimodal landscape, the proposed MOEA/PSL converges better than SparseEA and significantly outperforms LMEA, WOF-SMPSO, and MaOEA-IT. For SMOP8 with complex variable interactions, the difference between MOEA/PSL and SparseEA becomes larger. This is because SparseEA does not consider any variable interaction when generating offspring solutions, whereas the proposed MOEA/PSL can learn the variable interactions by RBM and DAE. As for SMOP5 with a unimodal landscape, both MOEA/PSL and SparseEA have satisfactory convergence performance, since the non-sparse region of the Pareto optimal solutions of SMOP5 is unfixed and quite easy to be detected. Moreover, according to the convergence profiles of IGD values obtained by the five MOEAs shown in Fig. 5, it is obvious that MOEA/PSL converges faster than the other MOEAs during the whole evolutionary process. To summarize, the proposed MOEA/PSL can effectively solve sparse LMOPs with various landscapes.

\section{Results on Real-World Problems}

Table IV shows the HV values obtained by the five compared MOEAs on eight sparse LMOPs in real-world 
TABLE IV

HV VAlues ObTained by LMEA, WOF-SMPSO, MAOEA-IT, SPARseEA, AND the Proposed MOEA/PSL ON FS1-FS3, IS1-IS3, NN1-NN3, CD1-CD3, CN1-CN3, SR1-SR3, PM1-PM3, AND PO1-PO3, WHERE THE BEST RESULT IN EACH ROW IS HIGHLIGHTED.

\begin{tabular}{|c|c|c|c|c|c|c|}
\hline Problem & Dec & LMEA & WOF-SMPSO & MaOEA-IT & SparseEA & MOEA/PSL \\
\hline FS1 & 800 & $2.6893 \mathrm{e}-1(0.00 \mathrm{e}+0)-$ & $8.8087 \mathrm{e}-1(5.65 \mathrm{e}-16)-$ & $2.8697 \mathrm{e}-1(0.00 \mathrm{e}+0)-$ & $9.8467 \mathrm{e}-1(4.52 \mathrm{e}-16)-$ & $9.8478 \mathrm{e}-1(3.39 \mathrm{e}-16)$ \\
\hline FS2 & 5966 & $4.8819 \mathrm{e}-1(2.26 \mathrm{e}-16)-$ & $9.5529 \mathrm{e}-1(0.00 \mathrm{e}+0)-$ & $6.2641 \mathrm{e}-1(3.39 \mathrm{e}-16)-$ & $9.8215 \mathrm{e}-1(4.52 \mathrm{e}-16)-$ & $9.8719 \mathrm{e}-1(5.04 \mathrm{e}-3)$ \\
\hline FS3 & 10000 & $3.3145 \mathrm{e}-1(5.65 \mathrm{e}-17)-$ & $8.9075 \mathrm{e}-1(4.52 \mathrm{e}-16)-$ & 3.7800e-1 (1.13e-16) - & $9.7262 \mathrm{e}-1(7.90 \mathrm{e}-16) \approx$ & $9.7443 e-1(7.60 e-3)$ \\
\hline IS? & 4177 & $4.6320 \mathrm{e}-1(1.59 \mathrm{e}-2)-$ & 7.4703e-1 (3.19e-3) - & $4.7467 \mathrm{e}-1(7.97 \mathrm{e}-3)-$ & 7.0593e-1 (9.11e-3) - & $8.0424 \mathrm{e}-1(2.42 \mathrm{e}-2)$ \\
\hline IS3 & 11055 & $5.3065 \mathrm{e}-1(3.04 \mathrm{e}-3)-$ & $9.6268 \mathrm{e}-1(1.35 \mathrm{e}-3) \approx$ & $6.7505 \mathrm{e}-1(8.71 \mathrm{e}-3)-$ & $9.5401 \mathrm{e}-1(7.10 \mathrm{e}-4)-$ & $9.6778 \mathrm{e}-1(7.31 \mathrm{e}-3)$ \\
\hline NN1 & 1181 & $3.3298 \mathrm{e}-1(1.53 \mathrm{e}-2)-$ & $4.7043 \mathrm{e}-1(1.31 \mathrm{e}-2)-$ & $3.1841 \mathrm{e}-1(9.69 \mathrm{e}-2)-$ & $9.0530 \mathrm{e}-1(1.73 \mathrm{e}-2)-$ & $9.2951 \mathrm{e}-1(3.99 \mathrm{e}-3)$ \\
\hline CD1 & 1133 & $9.9357 \mathrm{e}-2(2.10 \mathrm{e}-2)-$ & 6.9976e-1 (1.70e-3) - & $3.7719 \mathrm{e}-1(8.57 \mathrm{e}-3)-$ & $5.0006 \mathrm{e}-1(0.00 \mathrm{e}+0)-$ & $7.0540 \mathrm{e}-1(1.13 \mathrm{e}-16)$ \\
\hline $\mathrm{CD} 2$ & 4039 & $8.7164 \mathrm{e}-2(1.20 \mathrm{e}-2)-$ & $6.7661 \mathrm{e}-1(1.65 \mathrm{e}-3)-$ & $4.0942 \mathrm{e}-1(4.68 \mathrm{e}-3)-$ & $5.4546 \mathrm{e}-1(3.42 \mathrm{e}-3)-$ & $6.9660 \mathrm{e}-1(3.55 \mathrm{e}-3)$ \\
\hline CD3 & 9885 & $3.1492 \mathrm{e}-1(1.28 \mathrm{e}-3) \approx$ & $5.8850 \mathrm{e}-1(2.02 \mathrm{e}-3) \approx$ & $3.7066 \mathrm{e}-1(2.00 \mathrm{e}-3)-$ & $5.3839 \mathrm{e}-1(1.07 \mathrm{e}-3)-$ & $5.8784 \mathrm{e}-1(8.28 \mathrm{e}-4)$ \\
\hline CN1 & 1176 & $7.0620 \mathrm{e}-1(1.77 \mathrm{e}-2)-$ & $8.6239 \mathrm{e}-1(8.82 \mathrm{e}-3)-$ & $5.9259 \mathrm{e}-1(1.40 \mathrm{e}-2)-$ & $9.4455 e-1(2.72 e-3)-$ & $9.7206 \mathrm{e}-1(2.12 \mathrm{e}-3)$ \\
\hline $\mathrm{CN} 2$ & 4039 & $5.6216 \mathrm{e}-1(3.26 \mathrm{e}-2)-$ & $7.9818 \mathrm{e}-1(9.42 \mathrm{e}-3)-$ & $5.5264 \mathrm{e}-1(1.73 \mathrm{e}-2)-$ & $8.6601 \mathrm{e}-1(1.22 \mathrm{e}-2)-$ & $9.2460 \mathrm{e}-1$ (5.36e-3) \\
\hline $\mathrm{CN} 3$ & 9885 & $4.4994 \mathrm{e}-1(2.57 \mathrm{e}-3)-$ & $7.1083 \mathrm{e}-1(2.28 \mathrm{e}-3)-$ & $2.5489 \mathrm{e}-1(6.69 \mathrm{e}-2)-$ & $7.1141 \mathrm{e}-1(1.28 \mathrm{e}-3)-$ & $7.2132 \mathrm{e}-1(4.28 \mathrm{e}-4)$ \\
\hline SR1 & 1024 & $1.5091 \mathrm{e}-1(1.23 \mathrm{e}-2)-$ & $1.1669 \mathrm{e}-1(6.35 \mathrm{e}-3)-$ & $1.0888 \mathrm{e}-1(6.59 \mathrm{e}-3)-$ & $2.6363 \mathrm{e}-1(1.62 \mathrm{e}-2)-$ & $3.2374 \mathrm{e}-1(1.91 \mathrm{e}-2)$ \\
\hline PM3 & 10000 & $8.2645 \mathrm{e}-3(1.81 \mathrm{e}-18)-$ & $9.2739 \mathrm{e}-2(3.67 \mathrm{e}-4)-$ & $9.2836 \mathrm{e}-3(1.17 \mathrm{e}-3)-$ & $1.1023 e-1(5.13 e-4)+$ & $9.5855 \mathrm{e}-2(3.18 \mathrm{e}-4)$ \\
\hline PO1 & 1000 & $9.1432 e-2(9.57 e-5)-$ & $9.2197 \mathrm{e}-2(2.90 \mathrm{e}-4)-$ & $9.1671 \mathrm{e}-2(7.46 \mathrm{e}-5)-$ & $1.2368 \mathrm{e}-1(1.63 \mathrm{e}-3) \approx$ & $1.2367 \mathrm{e}-1(5.84 \mathrm{e}-4)$ \\
\hline $\mathrm{PO} 2$ & 5000 & $9.1120 \mathrm{e}-2(2.82 \mathrm{e}-5)-$ & $9.1244 \mathrm{e}-2(5.42 \mathrm{e}-5)-$ & $9.1120 e-2(1.53 e-5)-$ & $1.2380 \mathrm{e}-1(1.78 \mathrm{e}-3) \approx$ & $1.2412 \mathrm{e}-1(9.30 \mathrm{e}-4)$ \\
\hline $\mathrm{PO} 3$ & 10000 & $9.1082 e-2(3.97 e-5)-$ & $9.1097 \mathrm{e}-2(3.77 \mathrm{e}-5)-$ & $9.1041 \mathrm{e}-2(1.06 \mathrm{e}-5)-$ & $1.2429 \mathrm{e}-1(1.48 \mathrm{e}-3) \approx$ & $1.2481 \mathrm{e}-1(2.42 \mathrm{e}-4)$ \\
\hline$+/$ & & $0 / 23 / 1$ & $1 / 21 / 2$ & $0 / 24 / 0$ & $3 / 17 / 4$ & \\
\hline
\end{tabular}

' $+{ }^{\prime}, '{ }^{\prime}$ ' and ' $\approx$ ' indicate that the result is significantly better, significantly worse, and statistically similar to that obtained by the proposed MOEA/PSL, respectively.
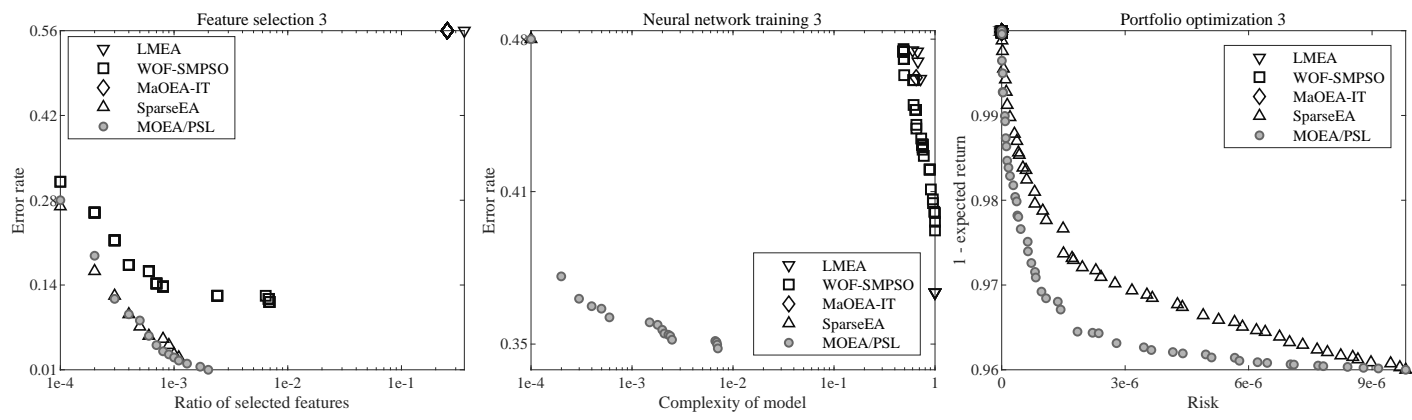

Fig. 6. Non-dominated solution sets with median HV obtained by LMEA, WOF-SMPSO, MaOEA-IT, SparseEA, and the proposed MOEA/PSL on FS3, NN3, and PO3 with approximately 10000 decision variables.
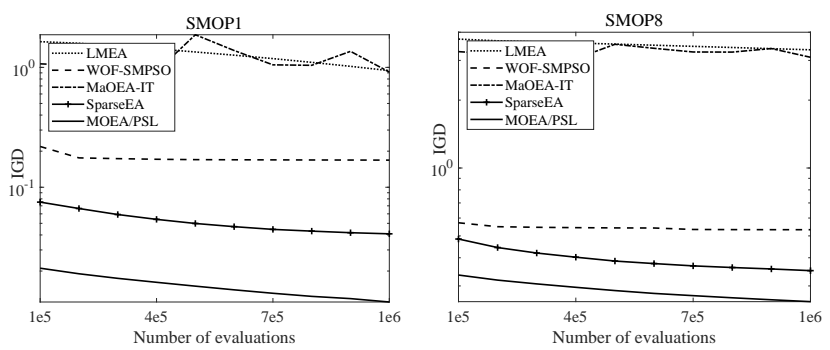

Fig. 5. Convergence profiles of IGD values obtained by LMEA, WOF-SMPSO, MaOEA-IT, SparseEA, and the proposed MOEA/PSL on SMOP1 and SMOP8 with 10000 decision variables.

applications with approximately 1000, 5000 and 10000 decision variables. It can be seen that the proposed MOEA/PSL obtains the best overall performance on these real-world problems, having achieved the best performance on 19 out of 24 test instances. Besides, SparseEA and WOF-SMOPSO obtain 4 and 1 best result, respectively. Furthermore, Fig. 6 depicts the nondominated solution sets with median HV values among 30 runs obtained by the five compared MOEAs on FS3, NN3, and PO3 with approximately 10000 decision variables. For the feature selection problem, the solutions obtained by MOEA/PSL are slightly better than those obtained by SparseEA and WOF-SMPSO, while LMEA and MaOEA-IT can only find a single solution with bad convergence. For the neural network training problem, the solutions obtained by MOEA/PSL have good convergence and diversity, the solutions obtained by LMEA and WOF-SMPSO are not well-converged, and MaOEA-IT and SparseEA can only find a single 
TABLE V

RUNTIMES (IN SECOND) OF LMEA, WOF-SMPSO, MAOEA-IT, SPARSEEA, AND THE PROPOSED MOEA/PSL ON BENCHMARK PROBLEMS AND REAL-WORLD PROBLEMS.

\begin{tabular}{|c|c|c|c|c|c|c|}
\hline Problem & Dec & LMEA & WOF-SMPSO & MaOEA-IT & SparseEA & MOEA/PSL \\
\hline $\begin{array}{l}\text { SMOP1- } \\
\text { SMOP8 } \\
\text { (average) }\end{array}$ & 10000 & $1.1042 \mathrm{e}+4$ & $5.6467 e+3$ & $8.2906 \mathrm{e}+3$ & $1.0391 \mathrm{e}+4$ & $6.8868 \mathrm{e}+3$ \\
\hline FS3 & 10000 & $2.4979 \mathrm{e}+4$ & $2.8925 e+3$ & $6.1546 \mathrm{e}+3$ & $5.4577 \mathrm{e}+2$ & $4.3347 e+2$ \\
\hline IS3 & 11055 & $2.1538 \mathrm{e}+5$ & $5.7815 e+5$ & $1.3433 \mathrm{e}+5$ & $4.2568 \mathrm{e}+4$ & $2.2611 \mathrm{e}+5$ \\
\hline NN3 & 10041 & $4.3060 \mathrm{e}+4$ & $9.3179 \mathrm{e}+3$ & $4.4536 \mathrm{e}+3$ & $4.8048 \mathrm{e}+3$ & $3.5246 e+3$ \\
\hline CD3 & 9885 & $1.2685 \mathrm{e}+5$ & $4.1383 \mathrm{e}+4$ & $1.3893 \mathrm{e}+5$ & $7.5853 \mathrm{e}+4$ & $6.1153 \mathrm{e}+4$ \\
\hline CN3 & 9885 & $5.7187 \mathrm{e}+4$ & $2.7179 e+4$ & $1.2290 \mathrm{e}+4$ & $3.5482 \mathrm{e}+4$ & $2.6756 e+4$ \\
\hline SR3 & 10240 & $3.7550 \mathrm{e}+4$ & $9.2712 \mathrm{e}+3$ & $7.5098 \mathrm{e}+3$ & $5.2169 e+3$ & $2.6068 \mathrm{e}+3$ \\
\hline PM3 & 10000 & $6.4473 e+4$ & $9.7101 \mathrm{e}+4$ & $6.3456 \mathrm{e}+3$ & $1.5784 \mathrm{e}+5$ & $1.1052 \mathrm{e}+5$ \\
\hline PO3 & 10000 & $8.1801 \mathrm{e}+3$ & $1.5218 \mathrm{e}+3$ & $1.9847 \mathrm{e}+3$ & $2.2545 \mathrm{e}+3$ & $2.2262 \mathrm{e}+3$ \\
\hline
\end{tabular}

solution. As for the portfolio optimization problem, the solutions obtained by MOEA/PSL have significantly better convergence than those obtained by SparseEA, and the solutions obtained by LMEA, WOF-SMPSO, and MaOEA-IT shrink to the upper-left corner of the objective space. As a consequence, these real-world problems are quite challenging for existing MOEAs, while the proposed MOEA/PSL is more promising for solving these problems than existing MOEAs.

In addition, the runtimes consumed by the five compared MOEAs on the eight benchmark problems and eight real-world problems with approximately 10000 decision variables are listed in Table V. It can be found that the runtime of MOEA/PSL is not significantly higher than the other MOEAs, though MOEA/PSL should train two neural networks at each generation. This is because the other MOEAs also have complex operations (e.g., variable interaction analysis in LMEA, Pareto-optimal subspace learning in MaOEA-IT, and population initialization in SparseEA), while the neural networks in MOEA/PSL are relatively small and easy to be trained. In short, the proposed MOEA/PSL has competitive efficiency to the other MOEAs.

\section{E. Effectiveness of the Two Neural Networks in MOEA/PSL}

The proposed MOEA/PSL learns the Pareto optimal subspace by both RBM and DAE, while a single neural network can also be used to achieve this goal. In this case, each solution does not need to be represented by a binary vector and a real vector, and the decision variables can directly be reduced. To verify the effectiveness of using both RBM and DAE, the proposed MOEA/PSL is compared with its variants using a single technique, including random embedding [60], PCA [61], RBM, and DAE. It is worth to note that although there exist many other machine learning techniques able to learn a subspace, the vectors reduced by most of them are not recoverable, hence they cannot be used in MOEA/PSL.

Table VI lists the IGD values obtained by MOEA/PSL and its four variants on SMOP1-SMOP8 with 1000 decision variables. Obviously, the original MOEA/PSL exhibits significantly better performance than those with a single technique on all the test problems. The superiority of using both RBM and DAE is mainly attributed to the consideration of sparsity, where the RBM is used to learn a sparsity distribution and the DAE is used to learn a compact representation. In short, it is necessary to learn the Pareto optimal subspace and consider the sparsity simultaneously in solving sparse LMOPs.

\section{F. Effectiveness of the Parameter Adaptation Strategy in} MOEA/PSL

There are two parameters related to the employment of RBM and DAE in MOEA/PSL, i.e., the ratio of offspring solutions generated in the Pareto optimal subspace $\rho$ and the size of the hidden layers $K$, both of which are adapted during the evolution of MOEA/PSL. To verify the effectiveness of the parameter adaptation strategy, MOEA/PSL is compared with some of its variants using a fixed $\rho$ or $K$.

Fig. 7 plots the convergence profiles of IGD and HV values obtained by MOEA/PSL with adaptive and fixed $\rho$ on SMOP7 with 1000 real variables and the instance selection problem with 862 binary variables. As shown in the figures, the MOEA/PSL with adaptive $\rho$ converges faster than the MOEA/PSL with $\rho=0.1, \rho=0.5$, and $\rho=1$. Besides, MOEA/PSL assigns different values to $\rho$ at different generations on different problems. Therefore, it is unreasonable to set $\rho$ to a fixed value; by contrast, adapting $\rho$ during the evolution of MOEA/PSL can lead to a relatively good performance on different problems. Fig. 8 plots the convergence profiles of IGD and HV values obtained by MOEA/PSL with adaptive and fixed $K$ on SMOP7 and the instance selection problem. Similarly, the MOEA/PSL with adaptive $K$ outperforms the MOEA/PSL with $K=10, K=20$, and $K=40$. To summarize, the parameter adaptation strategy can not only make MOEA/PSL parameterless, but also improve the performance in solving sparse LMOPs.

\section{CONCLUSIONS}

To address the limitations of existing MOEAs in solving LMOPs with sparse Pareto optimal solutions, this paper has proposed a Pareto optimal subspace learning based MOEA by using RBM and DAE. In each generation, an RBM is trained to learn a sparse distribution of the decision variables, and a DAE is trained to learn a compact representation. In this way, the decision variables can be reduced by the representation of the hidden layers of RBM and DAE. Moreover, a parameter adaptation strategy has been developed to determine the ratio of offspring solutions generated in the Pareto optimal subspace and the size of the hidden layers.

In the experiments, the proposed MOEA has been compared with four state-of-the-art MOEAs on eight benchmark problems and eight real-world problems taken from various fields. The experimental results have demonstrated that the proposed MOEA is more effective 
TABLE VI

IGD Values Obtained by MOEA/PSL and MOEA/PSL With a Single Technique on SMOP1-SMOP8, Where the Best Result in EACH ROW IS HighLIGHTED.

\begin{tabular}{cccccc}
\hline Problem & $\begin{array}{c}\text { MOEA/PSL } \\
\text { (only random embedding) }\end{array}$ & $\begin{array}{c}\text { MOEA/PSL } \\
\text { (only PCA) }\end{array}$ & $\begin{array}{c}\text { MOEA/PSL } \\
\text { (only RBM) }\end{array}$ & $\begin{array}{c}\text { MOEA/PSL } \\
\text { (only DAE) }\end{array}$ & MOEA/PSL \\
\hline SMOP1 & $9.5389 \mathrm{e}-2(5.56 \mathrm{e}-3)-$ & $3.1069 \mathrm{e}-1(5.95 \mathrm{e}-3)-$ & $1.3810 \mathrm{e}-1(1.32 \mathrm{e}-2)-$ & $3.4657 \mathrm{e}-1(8.12 \mathrm{e}-3)-$ & $1.1507 \mathrm{e}-2(2.11 \mathrm{e}-3)$ \\
SMOP2 & $3.6321 \mathrm{e}-1(3.41 \mathrm{e}-2)-$ & $9.5175 \mathrm{e}-1(2.24 \mathrm{e}-2)-$ & $1.5020 \mathrm{e}-1(4.21 \mathrm{e}-2)-$ & $9.7563 \mathrm{e}-1(2.62 \mathrm{e}-2)-$ & $4.2070 \mathrm{e}-2(9.70 \mathrm{e}-3)$ \\
SMOP3 & $1.1895 \mathrm{e}+0(3.61 \mathrm{e}-2)-$ & $1.1837 \mathrm{e}+0(3.90 \mathrm{e}-2)-$ & $2.2785 \mathrm{e}+0(2.05 \mathrm{e}-2)-$ & $1.2607 \mathrm{e}+0(2.87 \mathrm{e}-2)-$ & $1.2598 \mathrm{e}-2(3.06 \mathrm{e}-3)$ \\
SMOP4 & $6.1402 \mathrm{e}-2(1.01 \mathrm{e}-2)-$ & $4.2656 \mathrm{e}-1(1.59 \mathrm{e}-2)-$ & $5.9898 \mathrm{e}-2(6.25 \mathrm{e}-3)-$ & $4.3297 \mathrm{e}-1(1.29 \mathrm{e}-2)-$ & $4.7175 \mathrm{e}-3(2.47 \mathrm{e}-4)$ \\
SMOP5 & $3.9132 \mathrm{e}-1(3.36 \mathrm{e}-3)-$ & $4.0154 \mathrm{e}-1(8.31 \mathrm{e}-4)-$ & $1.2366 \mathrm{e}-1(1.77 \mathrm{e}-2)-$ & $4.1233 \mathrm{e}-1(1.30 \mathrm{e}-3)-$ & $7.4279 \mathrm{e}-3(4.60 \mathrm{e}-4)$ \\
SMOP6 & $6.5513 \mathrm{e}-2(3.66 \mathrm{e}-3)-$ & $9.0714 \mathrm{e}-2(3.72 \mathrm{e}-3)-$ & $7.3576 \mathrm{e}-2(5.39 \mathrm{e}-3)-$ & $9.0152 \mathrm{e}-2(3.29 \mathrm{e}-3)-$ & $7.4349 \mathrm{e}-3(5.34 \mathrm{e}-4)$ \\
SMOP7 & $2.4670 \mathrm{e}-1(2.04 \mathrm{e}-2)-$ & $2.1980 \mathrm{e}-1(1.02 \mathrm{e}-2)-$ & $3.5022 \mathrm{e}-1(6.44 \mathrm{e}-2)-$ & $2.2567 \mathrm{e}-1(5.81 \mathrm{e}-3)-$ & $5.5240 \mathrm{e}-2(3.62 \mathrm{e}-2)$ \\
SMOP8 & $1.1996 \mathrm{e}+0(8.21 \mathrm{e}-2)-$ & $1.2495 \mathrm{e}+0(4.09 \mathrm{e}-2)-$ & $3.7650 \mathrm{e}-1(9.77 \mathrm{e}-3)-$ & $1.2564 \mathrm{e}+0(1.95 \mathrm{e}-2)-$ & $2.2823 \mathrm{e}-1(4.59 \mathrm{e}-2)$ \\
\hline$+/-/ \approx$ & $0 / 8 / 0$ & $0 / 8 / 0$ & $0 / 8 / 0$ & & $0 / 8 / 0$ \\
\hline
\end{tabular}

' - ' indicates that the result is significantly worse than that obtained by MOEA/PSL.

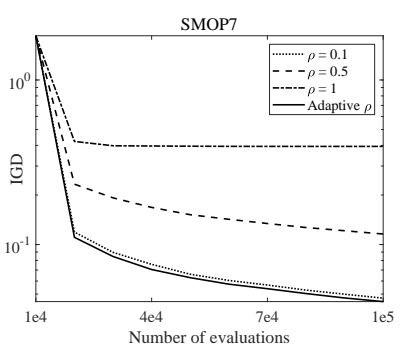

(a) IGD values on SMOP7.

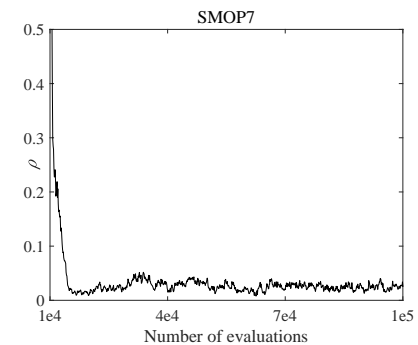

(b) Variation of $\rho$ on SMOP7.

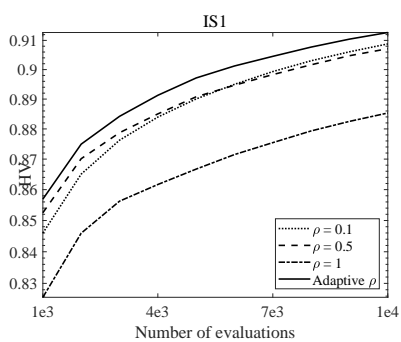

(c) HV values on IS1.

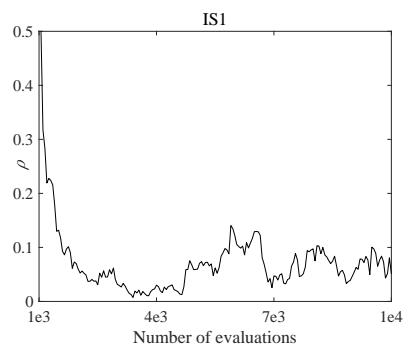

(d) Variation of $\rho$ on IS1.

Fig. 7. Convergence profiles of IGD and HV values obtained by MOEA/PSL with adaptive and fixed $\rho$ on SMOP7 and IS1.

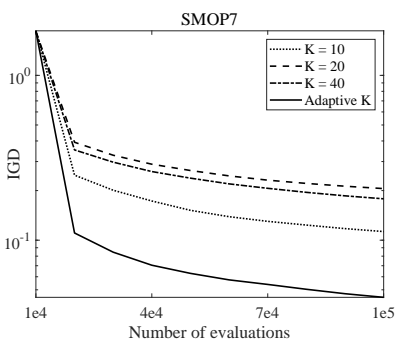

(a) IGD values on SMOP7.

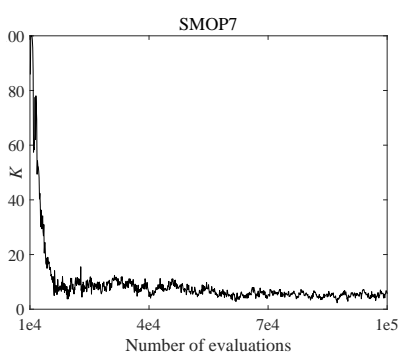

(b) Variation of $K$ on SMOP7.

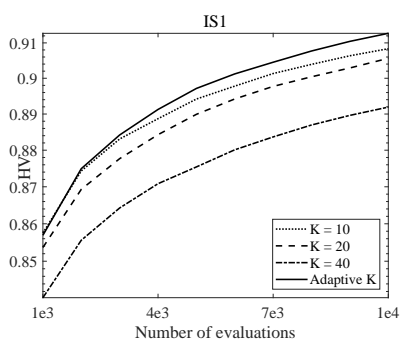

(c) HV values on IS1.

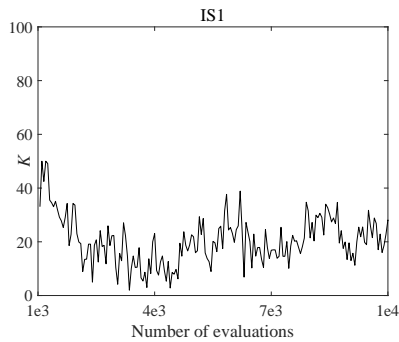

(d) Variation of $K$ on IS1.

Fig. 8. Convergence profiles of IGD and HV values obtained by MOEA/PSL with adaptive and fixed $K$ on SMOP7 and IS1.

than the compared MOEAs in solving sparse LMOPs with 1000 to 10000 decision variables.

This paper has revealed the necessity of Pareto optimal subspace learning in solving sparse LMOPs. Although the proposed MOEA has successfully achieved this goal by using RBM and DAE, it may become impractical when dealing with sparse problems having millions of decision variables (e.g., deep neural network training [62]), since the population is not large enough for training the neural networks, and the training process will be very time-consuming. Therefore, it is desirable to incorporate the proposed Pareto optimal subspace learning approach into other more effective MOEAs to solve super-large-scale problems.

\section{REFERENCES}

[1] J. Liu, M. Gong, Q. Miao, X. Wang, and H. Li, "Structure learning for deep neural networks based on multiobjective optimization,"
IEEE Transactions on Neural Networks and Learning Systems, vol. 29 no. 6, pp. 2450-2463, 2018.

[2] C. Cao, C. Li, Q. Yang, Y. Liu, and T. Qu, "A novel multi-objective programming model of relief distribution for sustainable disaster supply chain in large-scale natural disasters," Journal of Cleaner Production, vol. 174, pp. 1422-1435, 2018.

[3] S. Mouassa and T. Bouktir, "Multi-objective ant lion optimization algorithm to solve large-scale multi-objective optimal reactive power dispatch problem," International Journal for Computation and Mathematics in Electrical and Electronic Engineering, vol. 38, no. 1, pp. 304-324, 2019.

[4] L. M. Antonio and C. A. Coello Coello, "Use of cooperative coevolution for solving large scale multiobjective optimization problems," in Proceedings of the 2013 IEEE Congress on Evolutionary Computation, 2013, pp. 2758-2765.

[5] A. W. Iorio and X. Li, "A cooperative coevolutionary multiobjective algorithm using non-dominated sorting," in Proceedings of the 2004 Genetic and Evolutionary Computation Conference, 2004, pp. 537-548.

[6] K. Deb, A. Pratap, S. Agarwal, and T. Meyarivan, "A fast and elitist multi-objective genetic algorithm: NSGA-II," IEEE Transactions on Evolutionary Computation, vol. 6, no. 2, pp. 182-197, 2002.

[7] X. Ma, F. Liu, Y. Qi, X. Wang, L. Li, L. Jiao, M. Yin, and M. Gong, 
"A multiobjective evolutionary algorithm based on decision variable analyses for multiobjective optimization problems with largescale variables," IEEE Transactions on Evolutionary Computation, vol. 20, no. 2, pp. 275-298, 2016.

[8] X. Zhang, Y. Tian, R. Cheng, and Y. Jin, "A decision variable clustering-based evolutionary algorithm for large-scale manyobjective optimization," IEEE Transactions on Evolutionary Computation, vol. 22, no. 1, pp. 97-112, 2018.

[9] H. Xu, W. Zeng, D. Zhang, and X. Zeng, "Moea/hd: A multiobjective evolutionary algorithm based on hierarchical decomposition," IEEE Transactions on Cybernetics, vol. 49, no. 2, pp. 517-526, Feb 2019

[10] H. Xu, W. Zeng, X. Zeng, and G. G. Yen, "An evolutionary algorithm based on minkowski distance for many-objective optimization," IEEE Transactions on Cybernetics, vol. 49, no. 11, pp. 3968-3979, Nov 2019.

[11] H. Zille, H. Ishibuchi, S. Mostaghim, and Y. Nojima, "A framework for large-scale multi-objective optimization based on problem transformation," IEEE Transactions on Evolutionary Computation, vol. 22, no. 2, pp. 260-275, 2018.

[12] C. He, L. Li, Y. Tian, X. Zhang, R. Cheng, Y. Jin, and X. Yao, "Accelerating large-scale multi-objective optimization via problem reformulation," IEEE Transactions on Evolutionary Computation, 2019 , in press.

[13] Y. Tian, X. Zheng, X. Zhang, and Y. Jin, "Efficient large-scale multiobjective optimization based on a competitive swarm optimizer," IEEE Transactions on Cybernetics, vol. 2019, in press.

[14] M. Dellnitz, O. Schütze, and T. Hestermeyer, "Covering Pareto sets by multilevel subdivision techniques," Journal of Optimization Theory \& Applications, vol. 124, no. 1, pp. 113-136, 2005.

[15] Q. Zhang, A. Zhou, and Y. Jin, "RM-MEDA: A regularity modelbased multiobjective estimation of distribution algorithm," IEEE Transactions on Evolutionary Computation, vol. 12, no. 1, pp. 41-63, 2008.

[16] R. Cheng, Y. Jin, K. Narukawa, and B. Sendhoff, "A multiobjective evolutionary algorithm using Gaussian process based inverse modeling," IEEE Transactions on Evolutionary Computation, vol. 19, no. 6, pp. 838-856, 2015.

[17] T. M. Hamdani, J.-M. Won, A. M. Alimi, and F. Karray, "Multiobjective feature selection with NSGA-II," in International Conference on Adaptive and Natural Computing Algorithms, 2007, pp. 240-247.

[18] H. Li, Q. Zhang, J. Deng, and Z.-B. Xu, "A preference-based multiobjective evolutionary approach for sparse optimization," IEEE Transactions on Neural Networks and Learning Systems, vol. 29, no. 5, pp. 1716-1731, 2018.

[19] Y. Tian, S. Yang, L. Zhang, F. Duan, and X. Zhang, "A surrogateassisted multi-objective evolutionary algorithm for large-scale task-oriented pattern mining," IEEE Transactions on Emerging Topics in Computational Intelligence, vol. 3, no. 2, pp. 106-116, 2019.

[20] A. Fischer and C. Igel, "An introduction to restricted Boltzmann machines," in Proceedings of the Iberoamerican Congress on Pattern Recognition. Springer, 2012, pp. 14-36.

[21] P. Vincent, H. Larochelle, Y. Bengio, and P.-A. Manzagol, "Extracting and composing robust features with denoising autoencoders," in Proceedings of the 25th International Conference on Machine Learning, 2008, pp. 1096-1103.

[22] B. Xue, M. Zhang, and W. N. Browne, "Particle swarm optimization for feature selection in classification: A multi-objective approach," IEEE Transactions on Cybernetics, vol. 43, no. 6, pp. 1656-1671, 2013.

[23] B. Tran, B. Xue, and M. Zhang, "A new representation in PSO for discretization-based feature selection," IEEE Transactions on Cybernetics, vol. 48, no. 6, pp. 1733-1746, 2018.

[24] N. Verbiest, J. Derrac, C. Cornelis, S. García, and F. Herrera, "Evolutionary wrapper approaches for training set selection as preprocessing mechanism for support vector machines: Experimental evaluation and support vector analysis," Applied Soft Computing, vol. 38, pp. 10-22, 2016.

[25] Z. Lu, I. Whalen, V. Boddeti, Y. Dhebar, K. Deb, E. Goodman, and W. Banzhaf, "NSGA-NET: a multi-objective genetic algorithm for neural architecture search," arXiv preprint arXiv:1810.03522, 2018.

[26] X. Liu, Y. Luo, X. Zhang, and Q. Zhu, "A black-box attack on neural networks based on swarm evolutionary algorithm," arXiv preprint arXiv:1901.09892, 2019.
[27] C. Qian, Y. Yu, and Z.-H. Zhou, "Pareto ensemble pruning," in Proceedings of the Twenty-Ninth AAAI Conference on Artificial Intelligence, 2015.

[28] Y. Tian, S. Yang, and X. Zhang, "An evolutionary multiobjective optimization based fuzzy method for overlapping community detection," IEEE Transactions on Fuzzy Systems, 2019, in press.

[29] M. Lalou, M. A. Tahraoui, and H. Kheddouci, "The critical node detection problem in networks: A survey," Computer Science Review, vol. 28, pp. 92-117, 2018.

[30] J. Yang and J. Liu, "Influence maximization-cost minimization in social networks based on a multiobjective discrete particle swarm optimization algorithm," IEEE Access, vol. 6, pp. 2320-2329, 2018.

[31] Y. Xiang, Y. Zhou, Z. Zheng, and M. Li, "Configuring software product lines by combining many-objective optimization and SAT solvers," ACM Transactions on Software Engineering and Methodology, vol. 26, no. 4, p. 14, 2018

[32] X. Zhang, F. Duan, L. Zhang, F. Cheng, Y. Jin, and K. Tang, "Pattern recommendation in task-oriented applications: A multiobjective perspective [application notes]," IEEE Computational Intelligence Magazine, vol. 12, no. 3, pp. 43-53, 2017.

[33] K. Lwin, R. Qu, and G. Kendall, "A learning-guided multiobjective evolutionary algorithm for constrained portfolio optimization," Applied Soft Computing, vol. 24, pp. 757-772, 2014.

[34] A. Rahmani and S. MirHassani, "A hybrid firefly-genetic algorithm for the capacitated facility location problem," Information Sciences, vol. 283, pp. 70-78, 2014.

[35] E. Zitzler and L. Thiele, "Multiobjective evolutionary algorithms: A comparative case study and the strength Pareto approach," IEEE transactions on Evolutionary Computation, vol. 3, no. 4, pp. 257-271, 1999.

[36] J. Zhao, Y. Xu, F. Luo, Z. Dong, and Y. Peng, "Power system fault diagnosis based on history driven differential evolution and stochastic time domain simulation," Information Sciences, vol. 275, pp. 13-29, 2014.

[37] Y. Jin and B. Sendhoff, "Pareto-based multiobjective machine learning: An overview and case studies," IEEE Transactions on Systems, Man, and Cybernetics, Part C (Applications and Reviews), vol. 38, no. 3, pp. 397-415, 2008.

[38] L. Zhang, H. Pan, Y. Su, X. Zhang, and Y. Niu, "A mixed representation-based multiobjective evolutionary algorithm for overlapping community detection," IEEE Transactions on Cybernetics, vol. 47, no. 9, pp. 2703-2716, 2017.

[39] S. Wang and D. Zhao, "Power grid fault diagnosis based on immune clonal constrained multi-objective optimization method," Power System Technology, vol. 41, no. 12, pp. 4061-4068, 2017.

[40] H. Qian and Y. Yu, "Solving high-dimensional multi-objective optimization problems with low effective dimensions," in Proceedings of the Thirty-First AAAI Conference on Artificial Intelligence, 2017, pp. 875-881.

[41] Y. Sun, B. Xue, M. Zhang, and G. G. Yen, "A new two-stage evolutionary algorithm for many-objective optimization," IEEE Transactions on Evolutionary Computation, 2018.

[42] Y. Tian, X. Zhang, C. Wang, and Y. Jin, "An evolutionary algorithm for large-scale sparse multi-objective optimization problems," IEEE Transactions on Evolutionary Computation, 2019, in press.

[43] L. v. d. Maaten and E. Postma, "Dimensionality reduction: A comparative review," Tiburg Centre for Creative Computing TR 2009-005, Tech. Rep., 2009.

[44] G. E. Hinton, "Training products of experts by minimizing contrastive divergence," Neural Computation, vol. 14, no. 8, pp. 17711800, 2002.

[45] J. Zhai, S. Zhang, J. Chen, and Q. He, "Autoencoder and its various variants," in Proceedings of the 2018 IEEE International Conference on Systems, Man, and Cybernetics, 2018, pp. 415-419.

[46] Y. LeCun, Y. Bengio, and G. Hinton, "Deep learning," Nature, vol. 521, no. 7553, pp. 436-444, 2015.

[47] H. Tang, V. A. Shim, K. C. Tan, and J. Y. Chia, "Restricted Boltzmann machine based algorithm for multi-objective optimization," in Proceedings of the 2010 IEEE Congress on Evolutionary Computation, 2010.

[48] L. Feng, L. Zhou, J. Zhong, A. Gupta, Y. S. Ong, K. C. Tan, and A. K. Qin, "Evolutionary multitasking via explicit autoencoding," IEEE Transactions on Cybernetics, vol. 49, no. 9, pp. 3457-3470, 2019.

[49] M. D. McKay, R. J. Beckman, and W. J. Conover, "A comparison of three methods for selecting values of input variables in the analysis of output from a computer code," Technometrics, vol. 42, no. 1, pp. 55-61, 2000. 
[50] X. Zhang, Y. Tian, R. Cheng, and Y. Jin, "An efficient approach to non-dominated sorting for evolutionary multi-objective optimization," IEEE Transactions on Evolutionary Computation, vol. 19, no. 2, pp. 201-213, 2015.

[51] K. Deb and R. B. Agrawal, "Simulated binary crossover for continuous search space," Complex Systems, vol. 9, no. 4, pp. 115$148,1995$.

[52] K. Deb and M. Goyal, "A combined genetic adaptive search (GeneAS) for engineering design," Computer Science and Informatics, vol. 26, no. 4, pp. 30-45, 1996.

[53] Y. Tian, R. Cheng, X. Zhang, and Y. Jin, "PlatEMO: A MATLAB platform for evolutionary multi-objective optimization," IEEE Computational Intelligence Magazine, vol. 12, no. 4, pp. 73-87, 2017.

[54] R. A. Rossi and N. K. Ahmed, "The network data repository with interactive graph analytics and visualization," in Proceedings of the Twenty-Ninth AAAI Conference on Artificial Intelligence, 2015. [Online]. Available: http://networkrepository.com

[55] J. Liang, M. Gong, H. Li, C. Yue, and B. Qu, "Problem definitions and evaluation criteria for the cec special session on evolutionary algorithms for sparse optimization," Technical Report, Computational Intelligence Laboratory, Zhengzhou University, Zhengzhou, China, Report\# 2018001, 2018.

[56] R. Agrawal and R. Srikant, "Fast algorithms for mining association rules," in Proceedings of the International Conference on Very Large Data Bases, 1994, pp. 487-499.

[57] A. J. Nebro, J. J. Durillo, J. Garcia-Nieto, C. A. Coello Coello, F. Luna, and E. Alba, "SMPSO: A new PSO-based metaheuristic for multi-objective optimization," in Proceedings of the 2009 IEEE Symposium on Computational Intelligence in Multi-Criteria DecisionMaking, 2009, pp. 66-73.

[58] E. Zitzler, L. Thiele, M. Laumanns, C. M. Fonseca, and V. G. Da Fonseca, "Performance assessment of multiobjective optimizers: An analysis and review," IEEE Transactions on Evolutionary Computation, vol. 7, no. 2, pp. 117-132, 2003.

[59] L. While, P. Hingston, L. Barone, and S. Huband, "A faster algorithm for calculating hypervolume," IEEE Transactions on Evolutionary Computation, vol. 10, no. 1, pp. 29-38, 2006.

[60] Z. Wang, M. Zoghi, F. Hutter, D. Matheson, and N. D. Freitas, "Bayesian optimization in high dimensions via random embeddings," in Proceedings of the Twenty-Third International Joint Conference on Artificial Intelligence, 2013, pp. 1778-1784.

[61] P. Baldi and K. Hornik, "Neural networks and principal component analysis: Learning from examples without local minima," Neural Networks, vol. 2, no. 1, pp. 53-58, 1989.

[62] Y. Sun, G. G. Yen, and Z. Yi, "Evolving unsupervised deep neural networks for learning meaningful representations," IEEE Transactions on Evolutionary Computation, vol. 23, no. 1, pp. 89-103, 2019.

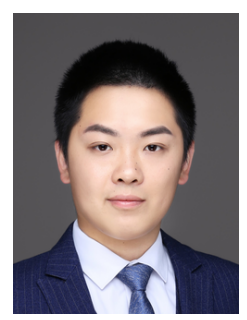

Ye Tian received the B.Sc., M.Sc., and Ph.D. degrees from Anhui University, Hefei, China, in 2012, 2015, and 2018, respectively.

He is currently an Associate Professor with the Institutes of Physical Science and Information Technology, Anhui University, Hefei, China, and also a Postdoctoral Research Fellow with the Department of Computer Science, City University of Hong Kong, Hong Kong. His current research interests include multi-objective optimization methods and their applications. He is the recipient of the 2018 IEEE Transactions on Evolutionary Computation Outstanding Paper Award and the 2020 IEEE Computational Intelligence Magazine Outstanding Paper Award.

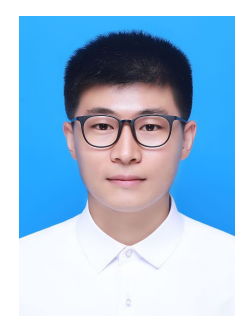

Chang $\mathrm{Lu}$ received the B.Sc. degree from Anhui University, Hefei, China, in 2017, where he is currently pursuing the M.Sc. degree with the School of Computer Science and Technology, Anhui University, Hefei, China.

His current research interests include multiobjective optimization and machine learning.

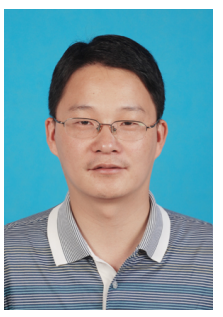

Xingyi Zhang (SM'18) received the B.Sc. degree from Fuyang Normal College, Fuyang, China, in 2003, and the M.Sc. and Ph.D. degrees from Huazhong University of Science and Technology, Wuhan, China, in 2006 and 2009, respectively.

He is currently a Professor with the School of Computer Science and Technology, Anhui University, Hefei, China. His current research interests include unconventional models and algorithms of computation, multi-objective optimization, and membrane computing. He is the recipient of the 2018 IEEE Transactions on Evolutionary Computation Outstanding Paper Award and the 2020 IEEE Computational Intelligence Magazine Outstanding Paper Award.

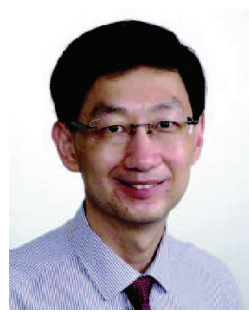

Kay Chen Tan (SM'08-F'14) received the B.Eng. (First Class Hons.) degree in electronics and electrical engineering and the Ph.D. degree from the University of Glasgow, Glasgow, U.K., in 1994 and 1997, respectively.

He is a Full Professor with the Department of Computer Science, City University of Hong Kong, Hong Kong. He has published over 200 refereed articles and six books.

Prof. Tan is the Editor-in-Chief of the IEEE TRANSACTIONS ON EVOLUTIONARY COMPUTATION, was the Editor-in-Chief of the IEEE Computational Intelligence Magazine from 2010 to 2013, and currently serves as the Editorial Board Member of over 10 journals. He is an elected member of the IEEE CIS AdCom from 2017 to 2019.

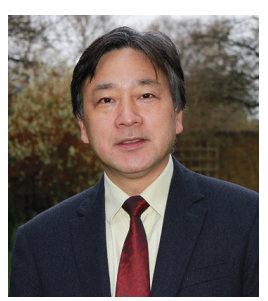

Yaochu Jin (SM'02-F'16) received the B.Sc., M.Sc., and Ph.D. degrees from Zhejiang University, Hangzhou, China, in 1988, 1991, and 1996, respectively, and the Dr.-Ing. degree from Ruhr University Bochum, Bochum, Germany, in 2001.

He is currently a Distinguished Chair Professor in Computational Intelligence, Department of Computer Science, University of Surrey, Guildford, U.K., where he heads the Nature Inspired Computing and Engineering Group. He was a Finland Distinguished Professor and a Changiiang Distinguished Visiting Professor. He has (co)authored over 300 peer-reviewed journal and conference papers and been granted eight patents on evolutionary optimization.

He is the Co-Editor-in-Chief of the IEEE Transactions on Cognitive and Developmental Systems and Complex \& Intelligent Systems. He is also an Associate Editor or Editorial Board Member of the IEEE Transactions on Evolutionary Computation, IEEE Transactions on Cybernetics, IEEE Transactions on Nanobioscience, Evolutionary Computation, BioSystems, Soft Computing, and Natural Computing. He is an IEEE Distinguished Lecturer (2017-2019). He is the recipient of the 2014 and 2016 IEEE Computational Intelligence Magazine Outstanding Paper Award, the 2018 IEEE Transactions on Evolutionary Computation Outstanding Paper Award, and the Best Paper Award of the 2010 IEEE Symposium on Computational Intelligence in Bioinformatics and Computational Biology. He is a Fellow of IEEE. 